TRANSACTIONS OF THE

AMERICAN MATHEMATICAL SOCIETY

Volume 360, Number 8, August 2008, Pages 4235-4262

S 0002-9947(08)04430-9

Article electronically published on February 27, 2008

\title{
BIMODULES AND $g$-RATIONALITY OF VERTEX OPERATOR ALGEBRAS
}

\author{
CHONGYING DONG AND CUIPO JIANG
}

\begin{abstract}
This paper studies the twisted representations of vertex operator algebras. Let $V$ be a vertex operator algebra and $g$ an automorphism of $V$ of finite order $T$. For any $m, n \in \frac{1}{T} \mathbb{Z}_{+}$, an $A_{g, n}(V)-A_{g, m}(V)$-bimodule $A_{g, n, m}(V)$ is constructed. The collection of these bimodules determines any admissible $g$-twisted $V$-module completely. A Verma type admissible $g$-twisted $V$-module is constructed naturally from any $A_{g, m}(V)$-module. Furthermore, it is shown with the help of bimodule theory that a simple vertex operator algebra $V$ is $g$-rational if and only if its twisted associative algebra $A_{g}(V)$ is semisimple and each irreducible admissible $g$-twisted $V$-module is ordinary.
\end{abstract}

\section{INTRODUCTION}

This paper deals with twisted representations of vertex operator algebras using the ideas of bimodules developed in DJ1-DJ3. The main result is a characterization of twisted rationality in terms of semisimplicity of certain associative algebras defined and studied in DLM2.

Twisted representations, which are also called twisted sectors or twisted modules, are the main ingredients in orbifold conformal field theory (see DHVW1DHVW2, [L1]-[L2, [FLM1-[FLM2, DVVV], DM], DLM0, HMT], DLM2], DLM4], DLM5, DY] and [MT]). The twisted sectors play a fundamental role in the construction of the moonshine vertex operator algebra $V^{\natural}$ FLM2 and other orbifold vertex operator algebras [DGM]. Although there is a lot of progress in the study of twisted sectors and orbifold conformal field theory, the semisimplicity of various twisted module categories has not been fully understood.

Let $V$ be a vertex operator algebra and $g$ an automorphism of finite order $T$. There are three different notions of $g$-twisted modules, that is, weak $g$-twisted modules, admissible $g$-twisted modules and ordinary $g$-twisted modules (see [FFR], D, DLM2 $)$. An ordinary $g$-twisted $V$-module is admissible and an admissible $g$-twisted $V$-module is a weak $g$-twisted $V$-module. They differ by some grading assumptions. The main axiom in these modules is the twisted Jacobi identity which was motivated by the twisted vertex operators studied in [L1]-[L2] and [FLM1]FLM2.

Received by the editors August 1, 2006.

2000 Mathematics Subject Classification. Primary 17B69.

The first author was supported by NSF grants, China NSF grant 10328102 and a Faculty research grant from the University of California at Santa Cruz.

The second author was supported by China NSF grant 10571119 . 
We call a vertex operator algebra $V g$-rational if the admissible $g$-twisted $V$ module category is semisimple. It is proved in DLM2 that if $V$ is $g$-rational, then there are only finitely many irreducible admissible $g$-twisted $V$-modules up to isomorphism and each irreducible admissible $g$-twisted $V$-module is ordinary. So the concept of $g$-rationality is an analogue of semisimplicity of associative algebras. In fact, in this paper, $g$-rationality will be understood in terms of the semisimplicity of an associative algebra $A_{g}(V)$ investigated in DLM2.

Stimulated by the $A(V)$-theory developed in [Z, an associative algebra $A_{g}(V)$ was defined and studied in DLM2. In order to state the connection between the twisted representation theory of $V$ and the representation theory of $A_{g}(V)$ let $M=\bigoplus_{n \in \frac{1}{T} \mathbb{Z}_{+}} M(n)$ be an admissible $g$-twisted $V$-module with $M(0) \neq 0$. Then $M(0)$ is an $A_{g}(V)$-module. Moreover, the map $M \rightarrow M(0)$ gives a one-to-one correspondence between the irreducible admissible $g$-twisted $V$-modules and simple $A_{g}(V)$-modules. These results reduce the classification of irreducible admissible $g$-twisted $V$-modules to the classification of simple $A_{g}(V)$-modules. So the classification of irreducible admissible $g$-twisted $V$-modules is settled at least theoretically.

The main purpose of this paper is to establish a relationship between the $g$ rationality of $V$ and the semisimplicity of $A_{g}(V)$. It has already been proved in DLM2 that $g$-rationality of $V$ implies the semisimplicity of $A_{g}(V)$. We prove in this paper that $V$ is $g$-rational if and only if $A_{g}(V)$ is semisimple and each irreducible admissible $g$-twisted $V$-module is ordinary. Note that $g$-rationality is an external condition on $V$. The new result essentially gives an internal characterization of $g$ rationality as $A_{g}(V)$ is a quotient of $V$ DLM2. In the case that $g=1$ this result has been obtained in DJ3.

The main idea comes from [DJ1-DJ3. The associative algebra $A_{g}(V)$ was generalized to associative algebras $A_{g, n}(V)$ for any $n \in \frac{1}{T} \mathbb{Z}_{+}$so that $A_{g, 0}(V)=$ $A_{g}(V)$ [DLM4]. For an admissible $g$-twisted $V$-module $M=\bigoplus_{n \in \frac{1}{T} \mathbb{Z}_{+}} M(n)$ with $M(0) \neq 0, M(m)$ is a module of $A_{g, n}(V)$ for $m \leq n$. So $A_{g, n}(V)$ gives more information on $M$ than $A_{g}(V)$. Most importantly, $V$ is $g$-rational if and only if $A_{g, n}(V)$ is semisimple for all $n \in \frac{1}{T} \mathbb{Z}_{+}$. Our approach is to prove that if $A_{g}(V)$ is semisimple, then $A_{g, n}(V)$ is semisimple for all $n$.

We first construct $A_{g, n}(V)-A_{g, m}(V)$-bimodules $A_{g, n, m}(V)$ (for $n, m \in \frac{1}{T} \mathbb{Z}_{+}$), which establishes a bridge between $A_{g}(V)$ and $A_{g, n}(V)$. These bimodules carry the semisimplicity information from $A_{g}(V)$ to all $A_{g, n}(V)$. From the point of view of representation theory, the $A_{g, n}(V)-A_{g, m}(V)$-bimodule $A_{g, n, m}(V)$ is a universal covering of the $A_{g, n}(V)-A_{g, m}(V)$-bimodule $\operatorname{Hom}_{\mathbb{C}}(M(m), M(n))$ for any admissible $g$-twisted $V$-module $M$. The importance of the construction of these bimodules is that it gives a concrete construction of the Verma type admissible $g$ twisted $V$-module $M(U)$ generated by an $A_{g, m}(V)$-module $U$ such that $M(U)(n)=$ $A_{g, n, m}(V) \otimes_{A_{g, m}(V)} U$ for all $n \in \frac{1}{T} \mathbb{Z}_{+}$. Using this construction we can prove that there is a natural invariant pairing between $M(U)$ and $M\left(U^{*}\right)$ such that the left radical of this pairing is exactly the maximal proper submodule of $M(U)$ if $U$ is irreducible. Then $M\left(U^{*}\right)$ is an admissible $g^{-1}$-twisted $V$-module as $U^{*}$ is an $A_{g^{-1}, m}(V)$-module instead of an $A_{g, m}(V)$-module. So this makes the admissible $g$-twisted module theory more comparable with the classical highest weight module theory for affine Lie algebras or the Virasoro algebra. With the help of this construction we can also prove that if $A_{g}(V)$ is semisimple, then the Verma type 
admissible $g$-twisted $V$-module $M(U)$ generated by an irreducible $A_{g}(V)$-module $U$ is irreducible. This is the key step in the proof of the main theorem.

Since the setting and most of the results in this paper are modelled on those in [DJ1 and DJ3], which deals with the case $g=1$, we omit a lot of details in this paper and refer the reader to DJ1] and [DJ3.

\section{The associative algebra $A_{g, n}(V)$}

Let $(V, Y, \mathbf{1}, \omega)$ denote, as usual, a vertex operator algebra as defined in FLM2] (see also [B]) and $g$ be an automorphism of $V$ of finite order $T$. Decompose $V$ into a direct sum of eigenspaces of $g$,

$$
V=\bigoplus_{r \in \mathbb{Z} / T \mathbb{Z}} V^{r}
$$

where $V^{r}=\left\{v \in V \mid g v=e^{-2 \pi i r / T} v\right\}$. We first review the notions of weak, admissible and ordinary $g$-twisted modules from [DLM2] (see also [FLM2, FFR] and D]).

Definition 2.1. A weak $g$-twisted $V$-module $M$ is a vector space equipped with a linear map

$$
\begin{array}{rlrl}
Y_{M}(\cdot, z): & & & \rightarrow(\text { End } M)\{z\} \\
& v \mapsto Y_{M}(v, z)=\sum_{n \in \mathbb{Q}} v_{n} z^{-n-1} \quad\left(v_{n} \in \operatorname{End} M\right)
\end{array}
$$

which satisfies the following conditions for all $0 \leq r \leq T-1, u \in V^{r}, v \in V, w \in M$ :

$$
\begin{array}{r}
z_{0}^{-1} \delta\left(\frac{z_{1}-z_{2}}{z_{0}}\right) Y_{M}\left(u, z_{1}\right) Y_{M}\left(v, z_{2}\right)-z_{0}^{-1} \delta\left(\frac{z_{2}-z_{1}}{-z_{0}}\right) Y_{M}\left(v, z_{2}\right) Y_{M}\left(u, z_{1}\right) \\
=z_{2}^{-1}\left(\frac{z_{1}-z_{0}}{z_{2}}\right)^{-r / T} \delta\left(\frac{z_{1}-z_{0}}{z_{2}}\right) Y_{M}\left(Y\left(u, z_{0}\right) v, z_{2}\right) .
\end{array}
$$

As mentioned in [DLM2] (see also [FLM2]), the twisted Jacobi identity is equivalent to the associativity formula

$$
\left(z_{0}+z_{2}\right)^{k+\frac{r}{T}} Y_{M}\left(u, z_{0}+z_{2}\right) Y_{M}\left(v, z_{2}\right) w=\left(z_{2}+z_{0}\right)^{k+\frac{r}{T}} Y_{M}\left(Y\left(u, z_{0}\right) v, z_{2}\right) w
$$

where $w \in M$ and $k$ is a nonnegative integer such that $z^{k+\frac{r}{T}} Y_{M}(u, z) w$ involves only nonnegative integral powers of $z$, and the commutator formula

$$
\begin{aligned}
& {\left[Y_{M}\left(u, z_{1}\right), Y_{M}\left(v, z_{2}\right)\right] } \\
= & \operatorname{Res}_{z_{0}} z_{2}^{-1}\left(\frac{z_{1}-z_{0}}{z_{2}}\right)^{-r / T} \delta\left(\frac{z_{1}-z_{0}}{z_{2}}\right) Y_{M}\left(Y\left(u, z_{0}\right) v, z_{2}\right) .
\end{aligned}
$$

Definition 2.2. An ordinary $g$-twisted $V$-module is a weak $g$-twisted $V$-module $M$ which carries a $\mathbb{C}$-grading induced by the spectrum of $L(0)$. That is,

$$
M=\bigoplus_{\lambda \in \mathbb{C}} M_{\lambda}
$$

where $M_{\ell}=\{w \in M \mid L(0) w=\ell w\}$, where $L(0)$ is a component operator of $Y_{M}(\omega, z)=\sum_{n \in \mathbb{Z}} L(n) z^{-n-2}$. Moreover we require that $M_{\ell}$ is finite dimensional and for fixed $\ell, M_{\frac{n}{T}+\ell}=0$ for all small enough integers $n$. 
Let $\mathbb{Z}_{+}$be the set of nonnegative integers.

Definition 2.3. An admissible $g$-twisted $V$-module is a weak $g$-twisted $V$-module $M$ which carries a $\frac{1}{T} \mathbb{Z}_{+}$-grading

$$
M=\bigoplus_{n \in \frac{1}{T} \mathbb{Z}_{+}} M(n)
$$

satisfying the following:

$$
v_{m} M(n) \subseteq M(n+\mathrm{wt} v-m-1)
$$

for homogeneous $v \in V, m \in \frac{1}{T} \mathbb{Z}_{+}$.

It is easy to show that an ordinary $g$-twisted $V$-module is admissible. If $g=1$ we get the weak, ordinary and admissible $V$-modules.

We say that $V$ is $g$-rational if every admissible $g$-twisted $V$-module is completely reducible. $V$ is called rational if $V$ is 1-rational. It is proved in DLM2 that if $V$ is $g$-rational, then there are only finitely many irreducible admissible $g$-twisted $V$-modules up to isomorphism and each irreducible admissible module is ordinary.

Next we present the $A_{g, n}(V)$-theory following [DLM4]. Fix $n=l+\frac{i}{T} \in \frac{1}{T} \mathbb{Z}_{+}$ with $l$ a nonnegative integer and $0 \leq i \leq T-1$. For $0 \leq r \leq T-1$, define $\delta_{i}(r)=1$ if $i \geq r$ and $\delta_{i}(r)=0$ if $i<r$. We also set $\delta_{i}(T)=0$. Let $O_{g, n}(V)$ be the linear span of all $u \circ_{g, n} v$ and $L(-1) u+L(0) u$ where for homogeneous $u \in V^{r}$ and $v \in V$,

$$
u \circ_{g, n} v=\operatorname{Res}_{z} Y(u, z) v \frac{(1+z)^{\mathrm{wt} u-1+\delta_{i}(r)+l+r / T}}{z^{2 l+\delta_{i}(r)+\delta_{i}(T-r)+1}} .
$$

We also define a second product $*_{g, n}$ on $V$ for $u \in V^{r}$ and $v$ as follows:

$$
u *_{g, n} v=\sum_{m=0}^{l}(-1)^{m}\left(\begin{array}{c}
m+l \\
l
\end{array}\right) \operatorname{Res}_{z} Y(u, z) \frac{(1+z)^{\mathrm{wt} u+l}}{z^{l+m+1}} v
$$

if $r=0$ and $u * g, n v=0$ if $r>0$.

Define the linear space $A_{g, n}(V)$ to be the quotient $V / O_{g, n}(V)$. Then $A_{g, 0}(V)=$ $A_{g}(V)$ has already been defined and studied in DLM2.

Remark 2.4. The definition of $u \circ_{g, n} v$ in DLM4] is not correct where it was defined as

$$
u \circ_{g, n} v=\operatorname{Res}_{z} Y(u, z) v \frac{(1+z)^{\mathrm{wt} u-1+\delta_{i}(r)+l+r / T}}{z^{2 l+\delta_{i}(r)+\delta_{i}(T-r)}}
$$

with $\delta_{i}(T)=1$. But the results and proofs in DLM4 remain valid.

Let $W$ be a weak $g$-twisted $V$-module and $m \in \frac{1}{T} \mathbb{Z}_{+}$. Following DLM4 we define

$\Omega_{m}(W)=\left\{w \in W \mid u_{\mathrm{wt} u-1+k} w=0\right.$, for all homogeneous $u \in V$ and $\left.k>m\right\}$.

The following theorem was obtained in DLM4.

Theorem 2.5. Let $V$ be a vertex operator algebra and $g$ an automorphism of $V$ of finite order $T$. Let $M=\bigoplus_{m \in \frac{1}{T} \mathbb{Z}_{+}} M(m)$ be an admissible $g$-twisted $V$-module. Let $n \in \frac{1}{T} \mathbb{Z}_{+}$. Then

(1) $A_{g, n}(V)$ is an associative algebra whose product is induced by $*_{g, n}$.

(2) The identity map on $V$ induces an algebra epimorphism from $A_{g, n}(V)$ to $A_{g, n-\frac{1}{T}}(V)$. 
(3) Let $W$ be a weak $g$-twisted $V$-module. Then $\Omega_{n}(W)$ is an $A_{g, n}(V)$-module such that $v+O_{g, n}(V)$ acts as $o(v)=v_{\mathrm{wt} v-1}$ for homogeneous $v$.

(4) Each $M(m)$ for $m \leq n$ is an $A_{g, n}(V)$-submodule of $\Omega_{n}(M)$. Furthermore, $M$ is irreducible if and only if each $M(n)$ is an irreducible $A_{g, n}(V)$-module.

(5) For any $A_{g, n}(V)$-module $U$ which cannot factor through $A_{g, n-\frac{1}{T}}(V)$ there is a unique Verma type admissible g-twisted $V$-module $\bar{M}(U)$ generated by $U$ so that $\bar{M}(U)(0) \neq 0$ and $\bar{M}(U)(n)=U$. Moreover, for any weak $g$-twisted $V$-module $W$ and any $A_{g, n}(V)$-module homomorphism $f$ from $U$ to $\Omega_{n}(W)$ there is a unique $V$-module homomorphism from $\bar{M}(U)$ to $W$ which extends $f$.

(6) $V$ is g-rational if and only if $A_{g, n}(V)$ are finite dimensional semisimple algebras for all $n \in \frac{1}{T} \mathbb{Z}_{+}$.

(7) If $V$ is g-rational, then there are only finitely many irreducible admissible $g$-twisted $V$-modules up to isomorphism and each irreducible admissible g-twisted $V$-module is ordinary.

(8) The linear map $\phi$ from $V$ to $V$ defined by $\phi(u)=e^{L(1)}(-1)^{L(0)} u$ for $u \in V$ induces an anti-isomorphism from $A_{g, n}(V)$ to $A_{g^{-1}, n}(V)$.

\section{3. $A_{g, n}(V)-A_{g, m}(V)$-BIMOdule $A_{g, n, m}(V)$}

Let $V=(V, Y, \mathbf{1}, \omega)$ be a vertex operator algebra, and let $g$ be an automorphism of $V$ of finite order $T$. This section is an extension of bimodule theory developed in DJ1 from the untwisted case to the twisted case. In particular we will construct an $A_{g, n}(V)-A_{g, m}(V)$-bimodule $A_{g, n, m}(V)$.

For $k \in \mathbb{Z}$, we denote the image of $k$ in $\mathbb{Z} / T \mathbb{Z}$ by $\bar{k}$. Without confusion, if $0 \leq k \leq T-1$, we sometimes also denote $\bar{k} \in \mathbb{Z} / T \mathbb{Z}$ by $k$. Let $m, p, n \in(1 / T) \mathbb{Z}_{+}$. Then $m=l_{1}+\left(i_{1} / T\right), p=l_{2}+\left(i_{2} / T\right), n=l_{3}+\left(i_{3} / T\right) \in(1 / T) \mathbb{Z}$ with $l_{1}, l_{2}, l_{3}$ three nonnegative integers and $0 \leq i_{1}, i_{2}, i_{3} \leq T-1$. In the following discussion, we always denote $m, n, p$ as above until further notice.

Recall the decomposition (2.1). For homogeneous $u \in V^{r}, v \in V$, define the product $*_{g, m, p}^{n}$ on $V$ as follows:

$$
\begin{aligned}
u *_{g, m, p}^{n} v= & \sum_{i=0}^{l_{2}}(-1)^{i}\left(\begin{array}{c}
l_{1}+l_{3}-l_{2}-1+\delta_{i_{1}}(r)+\delta_{i_{3}}(T-r)+i \\
i
\end{array}\right) \\
& \cdot \operatorname{Res}_{z} \frac{(1+z)^{\mathrm{wt} u-1+l_{1}+\delta_{i_{1}}(r)+r / T}}{z^{l_{1}+l_{3}-l_{2}+\delta_{i_{1}}(r)+\delta_{i_{3}}(T-r)+i}} Y(u, z) v
\end{aligned}
$$

if $\overline{i_{2}-i_{3}}=r$ and

$$
u *_{g, m, p}^{n} v=0
$$

otherwise.

If $n=p$, we denote $*_{g, m, p}^{n}$ by $\bar{*}_{g, m}^{n}$. In this case, $u{ }_{g, m}^{n} v=0$ if $r \neq 0$ and

$$
u \bar{*}_{g, m}^{n} v=\sum_{i=0}^{l_{3}}(-1)^{i}\left(\begin{array}{c}
l_{1}+i \\
i
\end{array}\right) \operatorname{Res}_{z} \frac{(1+z)^{\mathrm{wt} u+l_{1}}}{z^{l_{1}+i+1}} Y(u, z) v
$$

for $r=0$. One can easily check that $1{ }^{{ }^{n}}{ }_{g, m} u=u$, for $u \in V^{r}$. 
If $m=p$, we denote $*_{g, m, p}^{n}$ by $*_{g, m}^{n}$. In this case, $u *_{g, m}^{n} v=0$ if $\overline{i_{1}-i_{3}} \neq r$; if $\overline{i_{1}-i_{3}}=r$, then $-1+\delta_{i_{1}}(r)+\delta_{i_{3}}(T-r)=0$. So

$$
u *_{g, m}^{n} v=\sum_{i=0}^{l_{1}}(-1)^{i}\left(\begin{array}{c}
l_{3}+i \\
i
\end{array}\right) \operatorname{Res}_{z} \frac{(1+z)^{\mathrm{wt} u-1+l_{1}+\delta_{i_{1}}(r)+r / T}}{z^{l_{3}+i+1}} Y(u, z) v .
$$

If $g=1$, then $*_{g, m, p}^{n}$ is the same as $*_{m, p}^{n}$ defined in DJ1. If $m=p=n, *_{g, m, p}^{n}$ is just $*_{g, n}$ which has been defined in [DLM4] (see Section 2 of this paper). As in DLM4, we will denote the product by $*_{g, n}$ in this paper.

Let $O_{g, n, m}^{\prime}(V)$ be the linear span of $u \circ_{g, m}^{n} v$ and $(L(-1)+L(0)+m-n) u$, where for homogeneous $u \in V^{r}$ and $v \in V$,

$$
u \circ_{g, m}^{n} v=\operatorname{Res}_{z} \frac{(1+z)^{\mathrm{wt} u-1+\delta_{i_{1}}(r)+l_{1}+r / T}}{z^{l_{1}+l_{3}+\delta_{i_{1}}(r)+\delta_{i_{3}}(T-r)+1}} Y(u, z) v .
$$

Again if $m=n, u \circ_{g, m}^{n} v=u \circ_{g, n} v$ has been defined in Section 2 (see also [DLM4]). So if $m=n, O_{g, n, m}^{\prime}(V)=O_{g, n}(V)$.

Lemma 3.1. If $\overline{i_{1}-i_{3}} \neq r$, then $V^{r} \subseteq O_{g, n, m}^{\prime}(V)$.

Proof. Let $u \in V^{r}$ be homogeneous. Then $u \circ_{g, m}^{n} \mathbf{1} \in O_{g, n, m}^{\prime}(V)$. By the definition of $\circ_{g, m}^{n}$, we have

$$
\begin{aligned}
& u \circ_{g, m}^{n} \mathbf{1} \\
= & \sum_{j=0}^{\infty}\left(\begin{array}{c}
\mathrm{wt} u-1+\delta_{i_{1}}(r)+l_{1}+r / T \\
j
\end{array}\right) u_{j-l_{1}-l_{3}-\delta_{i_{1}}(r)-\delta_{i_{3}}(T-r)-1} \mathbf{1} \\
= & \sum_{j=0}^{l_{1}+l_{3}+\delta_{i_{1}}(r)+\delta_{i_{3}}(T-r)}\left(\begin{array}{c}
\mathrm{wt} u-1+\delta_{i_{1}}(r)+l_{1}+r / T \\
j
\end{array}\right) u_{j-l_{1}-l_{3}-\delta_{i_{1}}(r)-\delta_{i_{3}}(T-r)-1} \mathbf{1} .
\end{aligned}
$$

Using relations $u_{-s-1} \mathbf{1}=(1 / s !) L(-1)^{s} u$ for $s \geq 0$ and $L(-1) u \equiv(-L(0)-m+n) u$ modulo $O_{g, n, m}^{\prime}(V)$, we have

$$
\begin{aligned}
& u \circ_{g, m}^{n} \mathbf{1} \\
= & \sum_{j=0}^{k}\left(\begin{array}{c}
\mathrm{wt} u-1+\delta_{i_{1}}(r)+l_{1}+r / T \\
j
\end{array}\right)(-1)^{k-j} \\
& \cdot\left(\begin{array}{c}
\mathrm{wt} u+2 l_{1}+\delta_{i_{1}}(r)+\delta_{i_{3}}(T-r)-j-1+i_{1} / T-i_{3} / T \\
k-j
\end{array}\right) u \\
= & \frac{1}{k !}\left(\left(r / T-\left(i_{1}-i_{3}\right) / T\right)^{k}+\sum_{\substack{i, j \in \mathbb{Z}_{+} \\
0 \leq i+j<k}} a_{i, j}(r / T)^{i}\left(i_{1} / T-i_{3} / T\right)^{j}\right) u,
\end{aligned}
$$

where $k=l_{1}+l_{3}+\delta_{i_{1}}(r)+\delta_{i_{3}}(T-r)$ and $a_{i, j} \in \mathbb{Z}$. By the fact that $\overline{i_{1}-i_{3}} \neq r$, we know that $u \circ_{g, m}^{n} \mathbf{1} \equiv c u$ modulo $O_{g, n, m}^{\prime}(V)$ for a nonzero constant $c$. This shows $u \in O_{g, n, m}^{\prime}(V)$.

Corollary 3.2. Let $u \in V^{r}, v \in V^{s}$ be homogeneous. If $\overline{i_{1}-i_{2}} \neq s$, then $u *_{g, m, p}^{n} v \in$ $O_{g, n, m}^{\prime}(V)$. 
Proof. If $\overline{i_{2}-i_{3}} \neq r$, then $u *_{g, m, p}^{n} v=0$ by definition. If $\overline{i_{2}-i_{3}}=r$, then $u *_{g, m, p}^{n} v \in$ $V^{r+s}$ and $\overline{i_{1}-i_{3}} \neq \overline{r+s}$. The corollary follows from Lemma 3.1 .

The proof of the following lemma is fairly standard (cf. [DLM3 and [Z]).

Lemma 3.3. For homogeneous $u, v \in V$, and integers $k \geq s \geq 0$,

$$
\operatorname{Res}_{z} \frac{(1+z)^{\mathrm{wt} u-1+\delta_{i_{1}}(r)+l_{1}+r / T+s}}{z^{l_{1}+l_{3}+\delta_{i_{1}}(r)+\delta_{i_{3}}(T-r)+1+k}} Y(u, z) v \in O_{g, n, m}^{\prime}(V) .
$$

Lemma 3.4. For homogeneous $u \in V^{r}$ and $v \in V^{s}$, if $\overline{i_{2}-i_{3}}=r, \overline{i_{1}-i_{2}}=s$, and $m+n-p \geq 0$, then

$$
u *_{g, m, p}^{n} v-v *_{g, m, m+n-p}^{n} u-\operatorname{Res}_{z}(1+z)^{\mathrm{wt} u-1+p-n} Y(u, z) v \in O_{g, n, m}^{\prime}(V) .
$$

Proof. From the assumption that $\overline{i_{2}-i_{3}}=r$ and $\overline{i_{1}-i_{2}}=s$, one can easily deduce that $-1+\delta_{i_{1}}(s)+\delta_{i_{2}}(T-s)=0$ and $-1+\delta_{i_{1}}(r)+\delta_{i_{3}}(T-r)=\varepsilon$, where

$$
\varepsilon=\left\{\begin{array}{l}
1 \text { if } i_{1}+i_{3}-i_{2} \geq T \\
0 \text { if } 0 \leq i_{1}+i_{3}-i_{2}<T \\
-1 \quad \text { if } i_{1}+i_{3}-i_{2}<0
\end{array}\right.
$$

From the definition of $O_{g, n, m}^{\prime}(V)$, we have

$$
Y(v, z) u \equiv(1+z)^{-\mathrm{wt} u-\mathrm{wt} v-m+n} Y\left(u, \frac{-z}{1+z}\right) v
$$

modulo $O_{g, n, m}^{\prime}(V)$ (cf. [Z] and [DLM2]). Hence

$$
\begin{aligned}
& v *_{g, m, m+n-p}^{n} u \\
& =\sum_{i=0}^{l_{1}+l_{3}-l_{2}+\varepsilon}(-1)^{i}\left(\begin{array}{c}
l_{2}+i \\
i
\end{array}\right) \operatorname{Res}_{z} \frac{(1+z)^{\mathrm{wt} v-1+l_{1}+\delta_{i_{1}}(s)+s / T}}{z^{l_{2}+i+1}} Y(v, z) u \\
& \equiv \sum_{i=0}^{l_{1}+l_{3}-l_{2}+\varepsilon}(-1)^{i}\left(\begin{array}{c}
l_{2}+i \\
i
\end{array}\right) \operatorname{Res}_{z} \frac{(1+z)^{-\mathrm{wt} u-1+l_{3}+\delta_{i_{1}}(s)+\left(s-i_{1}+i_{3}\right) / T}}{z^{l_{2}+i+1}} Y\left(u, \frac{-z}{1+z}\right) v \\
& =\sum_{i=0}^{\left(\bmod O_{g, n, m}^{\prime}(V)\right)}(-1)^{l_{2}}\left(\begin{array}{c}
l_{2}+i \\
i
\end{array}\right) \operatorname{Res}_{z} \frac{(1+z)^{\mathrm{wt} u-1+l_{2}-l_{3}+i+\left(i_{2}-i_{3}\right) / T}}{z^{l_{2}+i+1}} Y(u, z) v .
\end{aligned}
$$

Recall the definition of $u *_{g, m, p}^{n} v$ :

$$
\begin{aligned}
u *_{g, m, p}^{n} v= & \sum_{i=0}^{l_{2}}(-1)^{i}\left(\begin{array}{c}
l_{1}+l_{3}-l_{2}+\varepsilon+i \\
i
\end{array}\right) \\
& \cdot \operatorname{Res}_{z} \frac{(1+z)^{\mathrm{wt} u-1+l_{1}+\delta_{i_{1}}(r)+r / T}}{z^{l_{1}+l_{3}-l_{2}+\varepsilon+i+1}} Y(u, z) v .
\end{aligned}
$$


Since $\left(i_{3}+r-i_{2}\right) / T=\delta_{i_{3}}(T-r)$, we have

$$
u *_{g, m, p}^{n} v-v *_{g, m, m+n-p}^{n} u \equiv \operatorname{Res}_{z} A_{l_{2}, l_{1}+l_{3}-l_{2}+\varepsilon}(z)(1+z)^{\mathrm{wt} u-1+p-n} Y(u, z) v
$$

modulo $O_{g, n, m}^{\prime}(V)$ where

$$
\begin{aligned}
& A_{l_{2}, l_{1}+l_{3}-l_{2}+\varepsilon}(z)=\sum_{i=0}^{l_{2}}(-1)^{i}\left(\begin{array}{c}
l_{1}+l_{3}-l_{2}+\varepsilon+i \\
i
\end{array}\right) \frac{(1+z)^{l_{1}+l_{3}-l_{2}+\varepsilon+1}}{z^{l_{1}+l_{3}-l_{2}+\varepsilon+i+1}} \\
& -\sum_{i=0}^{l_{1}+l_{3}-l_{2}+\varepsilon}(-1)^{l_{2}}\left(\begin{array}{c}
l_{2}+i \\
i
\end{array}\right) \frac{(1+z)^{i}}{z^{l_{2}+i+1}} .
\end{aligned}
$$

The lemma now follows from Proposition 5.1 of [DJ1].

By Lemma 3.4 and the fact that $\mathbf{1} \bar{*}_{g, m}^{n} u=u$, we have

Corollary 3.5. Let $u \in V^{r}$ be homogeneous. Then

$$
u *_{g, m}^{n} \mathbf{1}-u \in O_{g, n, m}^{\prime}(V) .
$$

Lemma 3.6. $V{ }_{g, m}^{n} O_{g, n, m}^{\prime}(V) \subseteq O_{g, n, m}^{\prime}(V), O_{g, n, m}^{\prime}(V) *_{g, m}^{n} V \subseteq O_{g, n, m}^{\prime}(V)$.

Proof. The proof is similar to that of Lemma 2.5 in [DJ1. Let $u \in V^{r}, v \in V^{s}$, $w \in V^{q}$ be homogeneous. By the definition of $\bar{*}_{g, m}^{n}$, we can assume that $r=0$. So

$$
\begin{aligned}
& u \bar{*}_{g, m}^{n}\left(v \circ_{g, m}^{n} w\right) \\
& \equiv \sum_{i=0}^{l_{3}}(-1)^{i}\left(\begin{array}{c}
l_{1}+i \\
i
\end{array}\right) \operatorname{Res}_{z_{1}} \frac{\left(1+z_{1}\right)^{\mathrm{wt} u+l_{1}}}{z_{1}^{l_{1}+i+1}} Y\left(u, z_{1}\right) \\
& \cdot \operatorname{Res}_{z_{2}} \frac{\left(1+z_{2}\right)^{\mathrm{wt} v-1+\delta_{i_{1}}(s)+l_{1}+s / T}}{z_{2}^{l_{1}+l_{3}+\delta_{i_{1}}(s)+\delta_{i_{3}}(T-s)+1}} Y\left(v, z_{2}\right) w \\
& -\sum_{i=0}^{l_{3}}(-1)^{i}\left(\begin{array}{c}
l_{1}+i \\
i
\end{array}\right) \operatorname{Res}_{z_{2}} \frac{\left(1+z_{2}\right)^{\mathrm{wt} v-1+\delta_{i_{1}}(s)+l_{1}+s / T}}{z_{2}^{l_{1}+l_{3}+\delta_{i_{1}}(s)+\delta_{i_{3}}(T-s)+1}} Y\left(v, z_{2}\right) \\
& \cdot \operatorname{Res}_{z_{1}} \frac{\left(1+z_{1}\right)^{\mathrm{wt} u+l_{1}}}{z_{1}^{l_{1}+i+1}} Y\left(u, z_{1}\right) w \\
& =\sum_{i=0}^{l_{3}}(-1)^{i}\left(\begin{array}{c}
l_{1}+i \\
i
\end{array}\right) \sum_{j \geq 0}\left(\begin{array}{c}
\mathrm{wt} u+l_{1} \\
j
\end{array}\right) \sum_{k=0}^{\infty}\left(\begin{array}{c}
-l_{1}-i-1 \\
k
\end{array}\right) \\
& \cdot \operatorname{Res}_{z_{2}} \operatorname{Res}_{z_{1}-z_{2}} \frac{\left(1+z_{2}\right)^{\mathrm{wt} u+2 l_{1}-j+\mathrm{wt} v-1+\delta_{i_{1}}(s)+s / T}\left(z_{1}-z_{2}\right)^{j+k}}{z_{2}^{2 l_{1}+i+2+k+l_{3}+\delta_{i_{1}}(s)+\delta_{i_{3}}(T-s)}} \\
& \cdot Y\left(Y\left(u, z_{1}-z_{2}\right) v, z_{2}\right) w \\
& =\sum_{i=0}^{l_{3}}(-1)^{i}\left(\begin{array}{c}
l_{1}+i \\
i
\end{array}\right) \sum_{j \geq 0}\left(\begin{array}{c}
\mathrm{wt} u+l_{1} \\
j
\end{array}\right) \sum_{k=0}^{\infty}\left(\begin{array}{c}
-l_{1}-i-1 \\
k
\end{array}\right) \\
& \cdot \operatorname{Res}_{z_{2}} \frac{\left(1+z_{2}\right)^{\mathrm{wt} u+2 l_{1}-j+\mathrm{wt} v-1+\delta_{i_{1}}(s)+s / T}}{z_{2}^{2 l_{1}+i+2+k+l_{3}+\delta_{i_{1}}(s)+\delta_{i_{3}}(T-s)}} Y\left(u_{j+k} v, z_{2}\right) w .
\end{aligned}
$$

Note that the weight of $u_{j+k} v$ is wtu + wt $v-j-k-1$. By Lemma 3.3 we see that $u \bar{*}_{g, m}^{n}\left(v \circ_{g, m}^{n} w\right)$ lies in $O_{g, n, m}^{\prime}(V)$. 
By the definition of $*_{g, m}^{n}$ and Corollary [3.2. $\left(v \circ_{g, m}^{n} w\right) *_{g, m}^{n} u \in O_{g, n, m}^{\prime}(V)$ if $\overline{i_{1}-i_{3}} \neq \overline{s+q}$ or $r \neq 0$. So we can assume that $\overline{i_{1}-i_{3}}=\overline{s+q}$ and $r=0$. By Lemma 3.4 we have

$$
\begin{aligned}
& u \bar{*}_{g, m}^{n}\left(v \circ_{g, m}^{n} w\right)-\left(v \circ_{g, m}^{n} w\right) *_{g, m}^{n} u \\
\equiv & \operatorname{Res}_{z}(1+z)^{\mathrm{wt} u-1} Y(u, z)\left(v \circ_{g, m}^{n} w\right) \\
= & \operatorname{Res}_{z_{1}}\left(1+z_{1}\right)^{\mathrm{wt} u-1} \operatorname{Res}_{z_{2}} \frac{\left(1+z_{2}\right)^{\mathrm{wt} v-1+\delta_{i_{1}}(s)+l_{1}+s / T}}{z_{2}^{l_{1}+l_{3}+\delta_{i_{1}}(s)+\delta_{i_{3}}(T-s)+1}} Y\left(u, z_{1}\right) Y\left(v, z_{2}\right) w \\
\equiv & \sum_{j \geq 0}\left(\begin{array}{c}
\mathrm{wt} u-1 \\
j
\end{array}\right) \operatorname{Res}_{z_{2}} \operatorname{Res}_{z_{1}-z_{2}} \frac{\left(1+z_{2}\right)^{\mathrm{wt} u-j+\mathrm{wt} v-2+\delta_{i_{1}}(s)+l_{1}+s / T}}{z_{2}^{l_{1}+l_{3}+\delta_{i_{1}}(s)+\delta_{i_{3}}(T-s)+1}} \\
& \cdot\left(z_{1}-z_{2}\right)^{j} Y\left(Y\left(u, z_{1}-z_{2}\right) v, z_{2}\right) w \\
= & \sum_{j \geq 0}\left(\begin{array}{c}
\mathrm{wt} u-1 \\
j
\end{array}\right) \operatorname{Res}_{z_{2}} \frac{\left(1+z_{2}\right)^{\mathrm{wt} u-j+\mathrm{wt} v-2+\delta_{i_{1}}(s)+l_{1}+s / T}}{z_{2}^{l_{1}+l_{3}+\delta_{i_{1}}(s)+\delta_{i_{3}}(T-s)+1}} Y\left(u_{j} v, z_{2}\right) w \\
\in & O_{g, n, m}^{\prime}(V) .
\end{aligned}
$$

This proves that $\left(v \circ \circ_{g, m}^{n} w\right) *_{g, m}^{n} u \in O_{g, n, m}^{\prime}(V)$.

Finally we deal with $(L(-1) u+(L(0)+m-n) u) *_{g, m}^{n} v$ and $v \bar{*}_{g, m}^{n}(L(-1) u+$ $(L(0)+m-n) u)$. As before we assume that $u \in V^{r}, v \in V^{s}$ are homogeneous, $\overline{i_{1}-i_{3}}=r$ and $s=0$. Then $-1+\delta_{i_{1}}(r)+\delta_{i_{3}}(T-r)=0$. So

$$
\begin{aligned}
& (L(-1) u+(L(0)+m-n) u) *_{g, m}^{n} v \\
= & \sum_{i=0}^{l_{1}}(-1)^{i}\left(\begin{array}{c}
l_{3}+i \\
i
\end{array}\right) \operatorname{Res}_{z} \frac{(1+z)^{\mathrm{wt} u+l_{1}+\delta_{i_{1}}(r)+r / T}}{z^{l_{3}+i+1}} Y(L(-1) u, z) v \\
& +(\mathrm{wt} u+m-n) \sum_{i=0}^{l_{1}}(-1)^{i}\left(\begin{array}{c}
l_{3}+i \\
i
\end{array}\right) \operatorname{Res}_{z} \frac{(1+z)^{\mathrm{wt} u-1+l_{1}+\delta_{i_{1}}(r)+r / T}}{z^{l_{3}+i+1}} Y(u, z) v \\
= & \sum_{i=0}^{l_{1}}(-1)^{i}\left(\begin{array}{c}
l_{3}+i \\
i
\end{array}\right)\left(-l_{3}-\delta_{i_{1}}(r)-\left(r+i_{3}-i_{1}\right) / T\right) \\
& \cdot \operatorname{Res}_{z} \frac{(1+z)^{\mathrm{wt} u-1+l_{1}+\delta_{i_{1}}(r)+r / T}}{z^{l_{3}+i+1}} Y(u, z) v \\
& +\sum_{i=0}^{l_{1}}(-1)^{i}\left(\begin{array}{c}
l_{3}+i \\
i
\end{array}\right)\left(l_{3}+i+1\right) \operatorname{Res}_{z} \frac{(1+z)^{\mathrm{wt} u+l_{1}+\delta_{i_{1}}(r)+r / T}}{z^{l_{3}+i+2}} Y(u, z) v \\
= & (-1)^{l_{1}}\left(l_{1}+l_{3}+1\right)\left(\begin{array}{c}
l_{1}+l_{3} \\
l_{1}
\end{array}\right) \operatorname{Res}_{z} \frac{(1+z)^{\mathrm{wt} u-1+l_{1}+\delta_{i_{1}}(r)+r / T}}{z^{l_{1}+l_{3}+2}} Y(u, z) v .
\end{aligned}
$$

Since $\delta_{i_{1}}(r)+\delta_{i_{3}}(T-r)+1=2$, it follows that the last expression is in $O_{g, n, m}^{\prime}(V)$. Thus $(L(-1) u+(L(0)+m-n) u) *_{g, m}^{n} v$ belongs to $O_{g, n, m}^{\prime}(V)$. We now turn to $v \bar{*}_{g, m}^{n}(L(-1)+L(0)+m-n) u$. By Lemma 3.4, we have

$$
\begin{aligned}
& v \bar{\aleph}_{g, m}^{n}(L(-1) u+(L(0)+m-n) u)-(L(-1) u+(L(0)+m-n) u) *_{g, m}^{n} v \\
\equiv & \operatorname{Res}_{z}(1+z)^{\mathrm{wt} v-1} Y(v, z)(L(-1) u+(L(0)+m-n) u) \\
= & \sum_{i \geq 0}\left(\begin{array}{c}
\mathrm{wt} v-1 \\
i
\end{array}\right) v_{i} L(-1) u+(\operatorname{wt} u+m-n) \sum_{i \geq 0}\left(\begin{array}{c}
\operatorname{wt} v-1 \\
i
\end{array}\right) v_{i} u
\end{aligned}
$$




$$
\begin{aligned}
= & L(-1) \sum_{i \geq 0}\left(\begin{array}{c}
\mathrm{wt} v-1 \\
i
\end{array}\right) v_{i} u+\sum_{i \geq 0}\left(\begin{array}{c}
\mathrm{wt} v-1 \\
i
\end{array}\right) i v_{i-1} u \\
& +(\mathrm{wt} u+m-n) \sum_{i \geq 0}\left(\begin{array}{c}
\mathrm{wt} v-1 \\
i
\end{array}\right) v_{i} u \\
= & L(-1) \sum_{i \geq 0}\left(\begin{array}{c}
\mathrm{wt} v-1 \\
i
\end{array}\right) v_{i} u+\sum_{i \geq 0}\left(\begin{array}{c}
\mathrm{wt} v-1 \\
i+1
\end{array}\right)(i+1) v_{i} u \\
& +(\operatorname{wt} u+m-n) \sum_{i \geq 0}\left(\begin{array}{c}
\mathrm{wt} v-1 \\
i
\end{array}\right) v_{i} u \\
= & \sum_{i \geq 0}\left(\begin{array}{c}
\mathrm{wt} v-1 \\
i
\end{array}\right)(L(-1)+\mathrm{wt} v-i-1+\mathrm{wt} u+m-n) v_{i} u \\
= & \sum_{i \geq 0}\left(\begin{array}{c}
\mathrm{wt} v-1 \\
i
\end{array}\right)\left(L(-1) v_{i} u+L(0) v_{i} u+(m-n) v_{i} u\right),
\end{aligned}
$$

which is in $O_{g, n, m}^{\prime}(V)$. So $v \bar{*}_{g, m}^{n}(L(-1) u+(L(0)+m-n) u) \in O_{g, n, m}^{\prime}(V)$, as desired.

Lemma 3.7. We have $\left(a{\aleph_{g, m}^{n}}^{n} b *_{g, m}^{n} c-a{\varlimsup_{g, m}^{n}}^{n}\left(b *_{g, m}^{n} c\right) \in O_{g, n, m}^{\prime}(V)\right.$ for homogeneous $a, b, c \in V$.

Proof. Let $a \in V^{r}, b \in V^{s}, c \in V^{l}$ be homogeneous. By the definition of $*_{g, m, p}^{n}$ and Lemma 3.6. $\left(a \bar{*}_{g, m}^{n} b\right) *_{g, m}^{n} c, a \bar{*}_{g, m}^{n}\left(b *_{g, m}^{n} c\right) \in O_{g, n, m}^{\prime}(V)$ if $r \neq 0$ or $\overline{i_{1}-i_{3}} \neq s$ or $l \neq 0$. So we can assume that $r=l=0$ and $\overline{i_{1}-i_{3}}=s$. So

$$
\begin{aligned}
& \left(a \bar{*}_{g, m}^{n} b\right) *_{g, m}^{n} c \\
= & \sum_{k=0}^{l_{1}}(-1)^{k}\left(\begin{array}{c}
l_{3}+k \\
k
\end{array}\right) \sum_{i=0}^{l_{3}}(-1)^{i}\left(\begin{array}{c}
l_{1}+i \\
i
\end{array}\right) \sum_{j \geq 0}\left(\begin{array}{c}
\mathrm{wt} a+l_{1} \\
j
\end{array}\right) \\
& \cdot \operatorname{Res}_{z} \frac{(1+z)^{\mathrm{wt} a+\mathrm{wt} b-1+2 l_{1}-j+i+\delta_{i_{1}}(s)+s / T}}{z^{l_{3}+k+1}} Y\left(a_{j-l_{1}-i-1} b, z\right) c \\
= & \sum_{k=0}^{l_{1}}(-1)^{k}\left(\begin{array}{c}
l_{3}+k \\
k
\end{array}\right) \sum_{i=0}^{l_{3}}(-1)^{i}\left(\begin{array}{c}
l_{1}+i \\
i
\end{array}\right) \operatorname{Res}_{z_{2}} \operatorname{Res}_{z_{1}-z_{2}}\left(z_{1}-z_{2}\right)^{-l_{1}-i-1} \\
& \cdot \frac{\left(1+z_{1}\right)^{\mathrm{wt} a+l_{1}}\left(1+z_{2}\right)^{\mathrm{wt} b-1+l_{1}+i+\delta_{i_{1}}(s)+s / T}}{z_{2}^{l_{3}+k+1}} Y\left(Y\left(a, z_{1}-z_{2}\right) b, z_{2}\right) c \\
= & \sum_{k=0}^{l_{1}}(-1)^{k}\left(\begin{array}{c}
l_{3}+k \\
k
\end{array}\right) \sum_{i=0}^{l_{3}}(-1)^{i}\left(\begin{array}{c}
l_{1}+i \\
i
\end{array}\right) \sum_{j \geq 0}\left(\begin{array}{c}
-l_{1}-i-1 \\
j
\end{array}\right) \\
& \cdot \operatorname{Res}_{z_{1}} \operatorname{Res}_{z_{2}} \frac{\left(1+z_{1}\right)^{\mathrm{wt} a+l_{1}}\left(1+z_{2}\right)^{\mathrm{wt} b-1+l_{1}+i+\delta_{i_{1}}(s)+s / T}\left(-z_{2}\right)^{j}}{z_{1}^{l_{1}+i+1+j} z_{2}^{l_{3}+k+1}} \\
& \cdot Y\left(a, z_{1}\right) Y\left(b, z_{2}\right) c
\end{aligned}
$$




$$
\begin{aligned}
& -\sum_{k=0}^{l_{1}}(-1)^{k}\left(\begin{array}{c}
l_{3}+k \\
k
\end{array}\right) \sum_{i=0}^{l_{3}}(-1)^{i}\left(\begin{array}{c}
l_{1}+i \\
i
\end{array}\right) \sum_{j \geq 0}\left(\begin{array}{c}
-l_{1}-i-1 \\
j
\end{array}\right) \\
& \cdot \operatorname{Res}_{z_{2}} \operatorname{Res}_{z_{1}} \frac{\left(1+z_{1}\right)^{\mathrm{wt} a+l_{1}}\left(1+z_{2}\right)^{\mathrm{wt} b-1+l_{1}+i+\delta_{i_{1}}(s)+s / T} z_{1}^{j}}{\left(-z_{2}\right)^{l_{1}+i+1+j} z_{2}^{l_{3}+k+1}} Y\left(b, z_{2}\right) Y\left(a, z_{1}\right) c \\
\equiv & a \bar{*}_{g, m}^{n}\left(b *_{g, m}^{n} c\right)+\sum_{k=0}^{l_{1}}(-1)^{k}\left(\begin{array}{c}
l_{3}+k \\
k
\end{array}\right) \sum_{i=0}^{l_{3}}(-1)^{i}\left(\begin{array}{c}
l_{1}+i \\
i
\end{array}\right) \\
& \cdot \operatorname{Res}_{z_{1}} \operatorname{Res}_{z_{2}}\left[\sum_{j=0}^{l_{3}-i}(-1)^{j}\left(\begin{array}{c}
-l_{1}-i-1 \\
j
\end{array}\right) \sum_{q=0}^{i}\left(\begin{array}{c}
i \\
q
\end{array}\right) \frac{z_{2}^{j+q}}{z_{1}^{j+i}}-\frac{1}{z_{1}^{i}}\right] \\
& \cdot \frac{\left(1+z_{1}\right)^{\mathrm{wt} a+l_{1}}\left(1+z_{2}\right)^{\mathrm{wt} b-1+l_{1}+\delta_{i_{1}}(s)+s / T}}{z_{1}^{l_{1}+1} z_{2}^{l_{3}+k+1}} Y\left(a, z_{1}\right) Y\left(b, z_{2}\right) c .
\end{aligned}
$$

The lemma then follows from Proposition 5.2 in DJ1].

Let $O_{g, n, m}^{\prime \prime}(V)$ be the linear span of

$$
u *_{g, m, p_{3}}^{n}\left(\left(a *_{g, p_{1}, p_{2}}^{p_{3}} b\right) *_{g, m, p_{1}}^{p_{3}} c-a *_{g, m, p_{2}}^{p_{3}}\left(b *_{g, m, p_{1}}^{p_{2}} c\right)\right),
$$

for all $a, b, c, u \in V$ and all $p_{1}, p_{2}, p_{3} \in \frac{1}{T} \mathbb{Z}_{+}$. Set

$$
O_{g, n, m}^{\prime \prime \prime}(V)=\sum_{p_{1}, p_{2} \in \frac{1}{T} \mathbb{Z}_{+}}\left(V *_{g, p_{1}, p_{2}}^{n} O_{g, p_{2}, p_{1}}^{\prime}(V)\right) *_{g, m, p_{1}}^{n} V
$$

and

$$
O_{g, n, m}(V)=O_{g, n, m}^{\prime}(V)+O_{g, n, m}^{\prime \prime}(V)+O_{g, n, m}^{\prime \prime \prime}(V) .
$$

Lemma 3.8. For any $m, n, p \in \frac{1}{T} \mathbb{Z}_{+}$, we have

$$
V *_{g, m, p}^{n} O_{g, p, m}(V) \subseteq O_{g, n, m}(V), O_{g, n, p}(V) *_{g, m, p}^{n} V \subseteq O_{g, n, m}(V) .
$$

In particular, $V \bar{*}_{g, m}^{n} O_{g, n, m}(V) \subseteq O_{g, n, m}(V), O_{g, n, m}(V) *_{g, m}^{n} V \subseteq O_{g, n, m}(V)$.

Proof. Note that $\mathbf{1} \bar{*}_{g, m}^{n} u=u$ for any $u \in V$. We have

$$
O_{g, n, p}^{\prime}(V) *_{g, m, p}^{n} V \subseteq\left(V{ }_{g, p}^{n} O_{g, n, p}^{\prime}(V)\right) *_{g, m, p}^{n} V \subseteq O_{g, n, m}^{\prime \prime \prime}(V)
$$

and

$$
\left(a *_{g, p_{1}, p_{2}}^{n} b\right) *_{g, m, p_{1}}^{n} c-a *_{g, m, p_{2}}^{n}\left(b *_{g, m, p_{1}}^{p_{2}} c\right) \in O_{g, n, m}^{\prime \prime}(V)
$$

for $a, b, c \in V$ and $p, p_{1}, p_{2} \in \frac{1}{T} \mathbb{Z}_{+}$. Thus

$$
V *_{g, m, p_{2}}^{n}\left(O_{g, p_{2}, p_{1}}^{\prime}(V) *_{g, m, p_{1}}^{p_{2}} V\right) \subseteq O_{g, n, m}(V) .
$$

Using Corollary 3.5 and the definition of $O_{g, n, m}^{\prime \prime \prime}(V)$ gives

(3.5) $V *_{g, m, p}^{n} O_{g, p, m}^{\prime}(V) \subseteq\left(V *_{g, m, p}^{n} O_{g, p, m}^{\prime}(V)\right) *_{g, m}^{n} V+O_{g, n, m}^{\prime}(V) \subseteq O_{g, n, m}(V)$.

By (3.2) and (3.5), it is enough to prove that

$$
V *_{g, m, p}^{n}\left(\left(V *_{g, p_{1}, p_{2}}^{p} O_{g, p_{2}, p_{1}}^{\prime}(V)\right) *_{g, m, p_{1}}^{p} V+O_{g, p, m}^{\prime \prime}(V)\right) \subseteq O_{g, n, m}(V)
$$

and

$$
\left(\left(V *_{g, p_{1}, p_{2}}^{n} O_{g, p_{2}, p_{1}}^{\prime}(V)\right) *_{g, p, p_{1}}^{n} V+O_{g, n, p}^{\prime \prime}(V)\right) *_{g, m, p}^{n} V \subseteq O_{g, n, m}(V)
$$

for $p_{1}, p_{2}, p \in \frac{1}{T} \mathbb{Z}_{+}$. 
We only prove (3.6). The proof of (3.7) is similar. By the definition of $O_{g, n, m}(V)$ and (3.3) and (3.4), we have

$$
\begin{aligned}
& V *_{g, m, p}^{n}\left(\left(V *_{g, p_{1}, p_{2}}^{p} O_{g, p_{2}, p_{1}}^{\prime}(V)\right) *_{g, m, p_{1}}^{p} V\right) \\
& \subseteq V *_{g, m, p}^{n}\left(V *_{g, m, p_{2}}^{p}\left(O_{g, p_{2}, p_{1}}^{\prime}(V) *_{g, m, p_{1}}^{p_{2}} V\right)\right)+O_{g, n, m}(V) \\
& \subseteq\left(V *_{g, p_{2}, p}^{n} V\right) *_{g, m, p_{2}}^{n}\left(O_{g, p_{2}, p_{1}}^{\prime}(V) *_{g, m, p_{1}}^{p_{2}} V\right)+O_{g, n, m}(V) \\
& \subseteq O_{g, n, m}(V) .
\end{aligned}
$$

It remains to prove that $V *_{g, m, p}^{n} O_{g, p, m}^{\prime \prime}(V) \subseteq O_{g, n, m}(V)$. By (3.3), we have

$$
\begin{aligned}
& v *_{g, m, p}^{n}\left(u *_{g, m, p_{3}}^{p}\left(\left(a *_{g, p_{1}, p_{2}}^{p_{3}} b\right) *_{g, m_{,} p_{1}}^{p_{3}} c-a *_{g, m, p_{2}}^{p_{3}}\left(b *_{g, m, p_{1}}^{p_{2}} c\right)\right)\right) \\
\equiv & \left(v *_{g, p_{3}, p}^{n} u\right) *_{g, m, p_{3}}^{n}\left(\left(a *_{g, p_{1}, p_{2}}^{p_{3}} b\right) *_{g, m, p_{1}}^{p_{3}} c-a *_{g, m, p_{2}}^{p_{3}}\left(b *_{g, m, p_{1}}^{p_{2}} c\right)\right) \\
\equiv & 0\left(\bmod O_{g, n, m}(V)\right) .
\end{aligned}
$$

We now define

$$
A_{g, n, m}(V)=V / O_{g, n, m}(V) .
$$

The reason for this definition will become clear from the $g$-twisted representation theory of $V$ discussed later. If $g=1$, the $A_{g, n, m}(V)=A_{n, m}(V)$ has been defined and studied in DJ1.

We have the first main theorem in this paper.

Theorem 3.9. $A_{g, n, m}(V)$ is an $A_{g, n}(V)-A_{g, m}(V)$-bimodule such that the left and right actions of $A_{g, n}(V)$ and $A_{g, m}(V)$ are given by ${ }_{g, m}^{n}$ and $*_{g, m}^{n}$.

\section{Properties of $A_{g, n, m}(V)$}

We will discuss some important properties of $A_{g, n, m}(V)$ in this section. As in DJ1, we will interpret these properties in terms of twisted representation theory in later sections. In fact, twisted representation theory is the origin of the bimodule $A_{g, n, m}(V)$ and its properties.

First we give an isomorphism between $A_{g, n, m}(V)$ and $A_{g^{-1}, m, n}(V)$ as $A_{g, n}(V)$ $A_{g, m}(V)$-bimodules. To achieve this we need to define actions of $A_{g, n}(V)$ and $A_{g, m}(V)$ on $A_{g^{-1}, m, n}(V)$ so that $A_{g^{-1}, m, n}(V)$ becomes an $A_{g, n}(V)-A_{g, m}(V)$-bimodule. Recall from [Z] the linear map $\phi: V \rightarrow V$ such that $\phi(v)=e^{L(1)}(-1)^{L(0)} v$ for $v \in V$. Then from Theorem 2.5 $\phi$ induces an anti-isomorphism from $A_{g, n}(V)$ to $A_{g^{-1}, n}(V)$.

Lemma 4.1. $A_{g^{-1}, m, n}(V)$ is an $A_{g, n}(V)-A_{g, m}(V)$-bimodule with the left action ${ }_{g, m}^{-n}$ of $A_{g, n}(V)$ and the right action ${ }_{g, m}^{n}$ of $A_{g, m}(V)$ defined by

$$
u_{g, m}^{-n} v=v *_{g^{-1}, n}^{m} \phi(u), \quad v \cdot_{g, m}^{n} w=\phi(w) \bar{*}_{g^{-1}, n}^{m} v
$$

for $v \in A_{g^{-1}, m, n}(V), u \in A_{g, n}(V), w \in A_{g, m}(V)$.

Proof. The proof is similar to that of Lemma 3.1 in [DJ1.

Proposition 4.2. The linear map $\phi: A_{g, n, m}(V) \rightarrow A_{g^{-1}, m, n}(V)$ defined by

$$
\phi(u)=e^{L(1)}(-1)^{L(0)} u,
$$


for $u \in A_{g, n, m}(V)$, is an $A_{g, n}(V)-A_{g, m}(V)$-bimodule isomorphism from $A_{g, n, m}(V)$ to $A_{g^{-1}, m, n}(V)$, where the actions of $A_{g, n}(V)$ and $A_{g, m}(V)$ on $A_{g, n, m}(V)$ are defined as in Theorem 3.9, and the actions on $A_{g^{-1}, m, n}(V)$ are defined as in Lemma 4.1.

Proof. Let $m=l_{1}+\left(i_{1} / T\right), p=l_{2}+\left(i_{2} / T\right), n=l_{3}+\left(i_{3} / T\right) \in \frac{1}{T} \mathbb{Z}$ with $l_{1}, l_{2}, l_{3}$ three nonnegative integers and $0 \leq i_{1}, i_{2}, i_{3} \leq T-1$.

We first prove that

$$
\phi\left(O_{g, n, m}^{\prime}(V)\right) \subset O_{g^{-1}, m, n}^{\prime}(V) .
$$

Recall the identities

$$
\begin{gathered}
(-1)^{L(0)} Y(u, z)(-1)^{L(0)}=Y\left((-1)^{L(0)} u,-z\right), \\
e^{L(1)} Y(u, z) e^{-L(1)}=Y\left(e^{(1-z) L(1)}(1-z)^{-2 L(0)} u, \frac{z}{1-z}\right)
\end{gathered}
$$

from [FHL].

Take $v=\operatorname{Res}_{z} \frac{(1+z)^{\mathrm{wt} a-1+\delta_{i_{1}}(r)+l_{1}+r / T}}{z^{l_{1}+l_{3}+\delta_{i_{1}}(r)+\delta_{i_{3}}(T-r)+1}} Y(a, z) b \in O_{g, n, m}^{\prime}(V)$, where $a \in V^{r}, b \in V$. Then

$$
\begin{aligned}
\phi(v)= & \operatorname{Res}_{z} e^{L(1)} \frac{(1+z)^{\mathrm{wt} a-1+\delta_{i_{1}}(r)+l_{1}+r / T}}{z^{l_{1}+l_{3}+\delta_{i_{1}}(r)+\delta_{i_{3}}(T-r)+1}} \\
\cdot & Y\left(e^{(1+z) L(1)}(1+z)^{-2 L(0)}(-1)^{L(0)} a, \frac{-z}{1+z}\right) e^{L(1)}(-1)^{L(0)} b \\
= & \operatorname{Res}_{z}(-1)^{\mathrm{wt} a+l_{1}+l_{3}+\delta_{i_{1}}(r)+\delta_{i_{3}}(T-r)+1} \frac{(1+z)^{\mathrm{wt} a+l_{3}+\delta_{i_{3}}(T-r)-r / T}}{z^{l_{1}+l_{3}+\delta_{i_{1}}(r)+\delta_{i_{3}}(T-r)+1}} \\
& \cdot Y\left(e^{\frac{1}{1+z} L(1)} a, z\right) e^{L(1)}(-1)^{L(0)} b \\
= & \operatorname{Res}_{z}(-1)^{\mathrm{wt} a+l_{1}+l_{3}+\delta_{i_{1}}(r)+\delta_{i_{3}}(T-r)+1} \sum_{j=0}^{\infty} \frac{1}{j !} \frac{(1+z)^{\mathrm{wt} a-j-1+l_{3}+\delta_{i_{3}}(T-r)+(T-r) / T}}{z^{l_{1}+l_{3}+\delta_{i_{1}}(r)+\delta_{i_{3}}(T-r)+1}} \\
& \cdot Y\left(L(1)^{j} a, z\right) e^{L(1)}(-1)^{L(0)} b,
\end{aligned}
$$

which is clearly in $O_{g^{-1}, m, n}^{\prime}(V)$ by the definition of $O_{g^{-1}, m, n}^{\prime}(V)$. For $u \in V^{r}$,

$$
\begin{aligned}
& \phi(L(-1) u+(L(0)+m-n) u) \\
= & e^{L(1)}(-1)^{L(0)} \operatorname{Res}_{z}(Y(\omega, z) u+z Y(\omega, z) u)+(m-n) e^{L(1)}(-1)^{L(0)} u \\
= & e^{L(1)} \operatorname{Res}_{z}(Y(\omega,-z)+z Y(\omega,-z))(-1)^{L(0)} u+(m-n) e^{L(1)}(-1)^{L(0)} u \\
= & \operatorname{Res}_{z}(1+z) Y\left(e^{(1+z) L(1)}(1+z)^{-2 L(0)}(-1)^{L(0)} \omega, \frac{-z}{1+z}\right) e^{L(1)}(-1)^{L(0)} u \\
& +(m-n) e^{L(1)}(-1)^{L(0)} u \\
= & \operatorname{Res}_{z}\left(-(1+z)^{2}+z(1+z)\right) Y\left(e^{(1+z)^{-1} L(1)} \omega, z\right) e^{L(1)}(-1)^{L(0)} u \\
& +(m-n) e^{L(1)}(-1)^{L(0)} u \\
= & -(L(-1)+L(0)) e^{L(1)}(-1)^{L(0)} u-(n-m) e^{L(1)}(-1)^{L(0)} u,
\end{aligned}
$$

which lies in $O_{g^{-1}, m, n}^{\prime}(V)$. So $\phi\left(O_{g, n, m}^{\prime}(V)\right) \subset O_{g^{-1}, m, n}^{\prime}(V)$.

We next prove that

$$
\phi\left(u *_{g, m, p}^{n} v\right) \equiv \phi(v) *_{g^{-1}, n, p}^{m} \phi(u)
$$

modulo $O_{g^{-1}, m, n}^{\prime}(V)$ for $u \in V^{r}, v \in V^{s}$. 
If $\overline{i_{2}-i_{3}} \neq r$, then $\overline{i_{3}-i_{2}} \neq T-r$. By the definition of $*_{g, m, p}^{n}$ and Corollary 3.2. we have $u *_{g, m, p}^{n} v=0$ and $\phi(v) *_{g^{-1}, n, p}^{m} \phi(u) \in O_{g^{-1}, m, n}^{\prime}(V)$, so (4.2) holds. Similarly, if $\overline{i_{1}-i_{2}} \neq s$, then $u *_{g, m, p}^{n} v \in O_{g, n, m}^{\prime}(V)$ and $\phi(v) *_{g^{-1}, n, p}^{m} \phi(u)=0$. In this case (4.2) follows from (4.1). So we assume that $\overline{i_{2}-i_{3}}=r, \overline{i_{1}-i_{2}}=s$. Then $-1+\delta_{i_{1}}(r)+\delta_{i_{3}}(T-r)=\varepsilon$, where $\varepsilon$ is defined as in (3.1). We have the following computation:

$$
\begin{aligned}
& \phi\left(u *_{g, m, p}^{n} v\right)=\phi\left(\sum_{i=0}^{l_{2}}(-1)^{i}\left(\begin{array}{c}
l_{1}+l_{3}-l_{2}-1+\delta_{i_{1}}(r)+\delta_{i_{3}}(T-r)+i \\
i
\end{array}\right)\right. \\
& \left.\cdot \operatorname{Res}_{z} \frac{(1+z)^{\mathrm{wt} u-1+l_{1}+\delta_{i_{1}}(r)+r / T}}{z^{l_{1}+l_{3}-l_{2}+\delta_{i_{1}}(r)+\delta_{i_{3}}(T-r)+i}} Y(u, z) v\right) \\
& =\sum_{i=0}^{l_{2}}(-1)^{i}\left(\begin{array}{c}
l_{1}+l_{3}-l_{2}+\varepsilon+i \\
i
\end{array}\right) \\
& \cdot \operatorname{Res}_{z} \frac{(1+z)^{\mathrm{wt} u-1+l_{1}+\delta_{i_{1}}(r)+r / T}}{z^{l_{1}+l_{3}-l_{2}+\varepsilon+i+1}} e^{L(1)} Y\left((-1)^{L(0)} u,-z\right)(-1)^{L(0)} v \\
& =\sum_{i=0}^{l_{2}}(-1)^{i}\left(\begin{array}{c}
l_{1}+l_{3}-l_{2}+\varepsilon+i \\
i
\end{array}\right) \operatorname{Res}_{z} \frac{(1+z)^{\mathrm{wt} u-1+l_{1}+\delta_{i_{1}}(r)+r / T}}{z^{l_{1}+l_{3}-l_{2}+\varepsilon+i+1}} \\
& \cdot Y\left(e^{(1+z) L(1)}(1+z)^{-2 L(0)}(-1)^{L(0)} u, \frac{-z}{1+z}\right) e^{L(1)}(-1)^{L(0)} v \\
& =\sum_{i=0}^{l_{2}}(-1)^{i+l_{1}+l_{3}-l_{2}+\varepsilon+\mathrm{wt} u}\left(\begin{array}{c}
l_{1}+l_{3}-l_{2}+\varepsilon+i \\
i
\end{array}\right) \\
& \cdot \operatorname{Res}_{z} \frac{(1+z)^{\mathrm{w} t u+l_{3}-l_{2}+i+\varepsilon-\delta_{i_{1}}(r)-r / T}}{z^{l_{1}+l_{3}-l_{2}+\varepsilon+i+1}} Y\left(e^{(1+z)^{-1} L(1)} u, z\right) e^{L(1)}(-1)^{L(0)} v \\
& =\sum_{j=0}^{\infty} \frac{1}{j !} \sum_{i=0}^{l_{2}}(-1)^{l_{1}+l_{3}-l_{2}+\varepsilon+\mathrm{wt} u}\left(\begin{array}{c}
l_{1}+l_{3}-l_{2}+\varepsilon+i \\
i
\end{array}\right) \\
& \cdot \operatorname{Res}_{z} \frac{(1+z)^{\mathrm{wt} u+l_{3}-l_{2}+i+\varepsilon-\delta_{i_{1}}(r)-r / T-j}}{z^{l_{1}+l_{3}-l_{2}+\varepsilon+i+1}} Y\left(L(1)^{j} u, z\right) e^{L(1)}(-1)^{L(0)} v \\
& =\sum_{j=0}^{\infty} \frac{(-1)^{\mathrm{w} t u}}{j !} \sum_{i=0}^{l_{1}+l_{3}-l_{2}+\varepsilon}(-1)^{i}\left(\begin{array}{c}
l_{2}+i \\
i
\end{array}\right) \\
& \cdot \operatorname{Res}_{z} \frac{(1+z)^{\mathrm{wt} u-1-j+l_{3}+\delta_{i_{3}}(T-r)+(T-r) / T}}{z^{l_{2}+i+1}} Y\left(L(1)^{j} u, z\right) e^{L(1)}(-1)^{L(0)} v \\
& -\sum_{j=0}^{\infty} \frac{(-1)^{\mathrm{wt} u}}{j !} \operatorname{Res}_{z}(1+z)^{\mathrm{wt} u-j-1+n-p} Y\left(L(1)^{j} u, z\right) e^{L(1)}(-1)^{L(0)} v \\
& \equiv \phi(v) *_{g^{-1}, n, p}^{m} \phi(u)\left(\bmod O_{g^{-1}, m, n}^{\prime}(V)\right),
\end{aligned}
$$

where we have used Proposition 5.1 of [DJ1 and Lemma 3.4] in the last two steps. In particular, $\phi\left(u \bar{*}_{g, m}^{n} v\right) \equiv \phi(v) *_{g^{-1}, n}^{m} \phi(u)$ modulo $O_{g^{-1}, m, n}^{\prime}(V)$ and $\phi\left(u *_{g, m}^{n} v\right) \equiv$ $\phi(v) \bar{*}_{g^{-1}, n}^{m} \phi(u)$ modulo $O_{g^{-1}, m, n}^{\prime}(V)$ for $u, v \in V$.

As in the proof of Proposition 3.2 in DJ1, we can easily deduce that $\phi\left(O_{g, n, m}(V)\right)$ $\subseteq O_{g^{-1}, m, n}(V)$ by using (4.1) and (4.2). Thus $\phi: A_{g, n, m}(V) \rightarrow A_{g^{-1}, m, n}(V)$ is a well-defined bimodule isomorphism. 
Proposition 4.3. Let $m, n, l \in \frac{1}{T} \mathbb{Z}_{+}$such that $m-l, n-l$ are nonnegative. Then $A_{g, n-l, m-l}(V)$ is an $A_{g, n}(V)-A_{g, m}(V)$-bimodule and the identity map on $V$ induces an epimorphism of $A_{g, n}(V)-A_{g, m}(V)$-bimodules from $A_{g, n, m}(V)$ to $A_{g, n-l, m-l}(V)$.

Proof. It is good enough to prove the result for $l=1 / T$. First, from the definition of $O_{g, n, m}^{\prime}(V)$ and Lemma 3.3, we can easily see that $O_{g, n, m}^{\prime}(V) \subseteq O_{g, n-1 / T, m-1 / T}^{\prime}(V)$.

Next, we prove that

$$
u *_{g, p_{1}, p_{2}}^{p_{3}} v \equiv u *_{g, p_{1}-1 / T, p_{2}-1 / T}^{p_{3}-1 / T} v\left(\bmod O_{g, p_{3}-1 / T, p_{1}-1 / T}^{\prime}(V)\right),
$$

for $p_{1}, p_{2}, p_{3} \in \frac{1}{T} \mathbb{Z}_{+}$.

Let $u \in V^{r}, v \in V^{s}$ be homogeneous and $p_{i}=s_{i}+j_{i} / T, i=1,2,3$. We can assume that $\overline{j_{2}-j_{3}}=r$ and $\overline{j_{1}-j_{2}}=s$.

We first assume that $r \neq 0$ and $j_{i} \neq 0, i=1,2,3$. Then $j_{3} \neq T-r$. It is easy to see that $\delta_{j_{1}}(r)=\delta_{j_{1}-1}(r)+\delta_{j_{1}, r}$ and $\delta_{j_{3}}(T-r)=\delta_{j_{3}-1}(T-r)+\delta_{j_{3}, T-r}$. So

$$
\begin{aligned}
u *_{g, p_{1}, p_{2}}^{p_{3}} v= & \sum_{i=0}^{s_{2}}(-1)^{i}\left(\begin{array}{c}
s_{1}+s_{3}-s_{2}-1+\delta_{j_{1}-1}(r)+\delta_{j_{1}, r}+\delta_{j_{3}-1}(T-r)+i \\
i
\end{array}\right) \\
& \cdot \operatorname{Res}_{z} \frac{(1+z)^{\mathrm{wt} u-1+s_{1}+\delta_{j_{1}-1}(r)+\delta_{j_{1}, r}+r / T}}{z^{s_{1}+s_{3}-s_{2}+\delta_{j_{1}-1}(r)+\delta_{j_{1}, r}+\delta_{j_{3}-1}(T-r)+i}} Y(u, z) v .
\end{aligned}
$$

If $j_{1} \neq r$, it is clear that $u *_{g, p_{1}, p_{2}}^{p_{3}} v=u *_{g, p_{1}-1 / T, p_{2}-1 / T}^{p_{3}-1 / T} v$. If $j_{1}=r$, then

$$
\begin{aligned}
& u *_{g, p_{1}, p_{2}}^{p_{3}} v=\sum_{i=0}^{s_{2}}(-1)^{i}\left(\begin{array}{c}
s_{1}+s_{3}-s_{2}+\delta_{j_{3}-1}(T-r)+i \\
i
\end{array}\right) \\
& \cdot \operatorname{Res}_{z} \frac{(1+z)^{\mathrm{wt} u+s_{1}+r / T}}{z^{s_{1}+s_{3}-s_{2}+\delta_{j_{3}-1}(T-r)+i+1}} Y(u, z) v \\
& =\sum_{i=0}^{s_{2}}\left(\begin{array}{c}
s_{1}+s_{3}-s_{2}+\delta_{j_{3}-1}(T-r)+i \\
i
\end{array}\right)(-1)^{i} \\
& \cdot \operatorname{Res}_{z} \frac{(1+z)^{\mathrm{wt} u+s_{1}+r / T-1}}{z^{s_{1}+s_{3}-s_{2}+\delta_{j_{3}-1}(T-r)+i}} Y(u, z) v \\
& +\sum_{i=0}^{s_{2}}\left(\begin{array}{c}
s_{1}+s_{3}-s_{2}+\delta_{j_{3}-1}(T-r)+i \\
i
\end{array}\right)(-1)^{i} \\
& \cdot \operatorname{Res}_{z} \frac{(1+z)^{\mathrm{wt} u+s_{1}+r / T-1}}{z^{s_{1}+s_{3}-s_{2}+\delta_{j_{3}-1}(T-r)+i+1}} Y(u, z) v \\
& \equiv \sum_{i=0}^{s_{2}}\left(\begin{array}{c}
s_{1}+s_{3}-s_{2}-1+\delta_{j_{3}-1}(T-r)+i \\
i
\end{array}\right)(-1)^{i} \\
& \cdot \operatorname{Res}_{z} \frac{(1+z)^{\mathrm{wt} u+s_{1}+r / T-1}}{z^{s_{1}+s_{3}-s_{2}+\delta_{j_{3}-1}(T-r)+i}} Y(u, z) v \quad\left(\bmod O_{g, p_{1}-1 / T, p_{3}-1 / T}^{\prime}(V)\right) \\
& =u *{ }_{g, p_{1}-1 / T, p_{2}-1 / T}^{p_{3}-1 / T} v \text {. }
\end{aligned}
$$

The proof of (4.3) for other cases is similar. Using (4.3), Lemma 3.8 and the definition of $O_{g, n, m}(V)$, we conclude that $O_{g, n, m}^{\prime \prime}(V), O_{g, n, m}^{\prime \prime \prime}(V) \subset O_{g, n-1 / T, m-1 / T}(V)$. This together with (4.3) finishes the proof. 
Similar to Proposition 3.4 in DJ1, we have the following result on tensor products of bimodules.

Proposition 4.4. Define the linear map $\varphi: A_{g, n, p}(V) \otimes_{A_{g, p}(V)} A_{g, p, m}(V) \rightarrow$ $A_{g, n, m}(V)$ by

$$
\varphi(u \otimes v)=u *_{g, m, p}^{n} v,
$$

for $u \otimes v \in A_{g, n, p}(V) \otimes_{A_{g, p}(V)} A_{g, p, m}(V)$. Then $\varphi$ is an $A_{g, n}(V)-A_{g, m}(V)$-bimodule homomorphism from $A_{g, n, p}(V) \otimes_{A_{g, p}(V)} A_{g, p, m}(V)$ to $A_{g, n, m}(V)$.

\section{TWISTED REPRESENTATION THEORY}

Let $M=\bigoplus_{n \in \frac{1}{T} \mathbb{Z}_{+}} M(n)$ be an admissible $g$-twisted $V$-module such that $M(0) \neq$ 0 . For homogeneous $u \in V^{r}$, and $m=l_{1}+i_{1} / T, n=l_{2}+i_{2} / T$ such that $l_{1}, l_{2} \in \mathbb{Z}_{+}$, $0 \leq i_{1}, i_{2} \leq T-1$, define the linear map $o_{g, n, m}(u): M(m) \rightarrow M(n)$ by

$$
o_{g, n, m}(u) w=u_{\mathrm{wt} u+m-n-1} w,
$$

where $w \in M(m)$ and $u_{\mathrm{wt} u+m-n-1}$ is the component operator of

$$
Y_{M}(u, z)=\sum_{n \in r / T+\mathbb{Z}} u_{n} z^{-n-1} .
$$

Note that if $r \neq \overline{i_{1}-i_{2}}$, then $o_{g, n, m}(u) w=0$.

Lemma 5.1. Let $a \in V^{r}, b \in V^{s}, m=l_{1}+\frac{i_{1}}{T}, p=l_{2}+\frac{i_{2}}{T}, n=l_{3}+\frac{i_{3}}{T} \in \frac{1}{T} \mathbb{Z}_{+}$ with $l_{1}, l_{2}, l_{3}$ three nonnegative integers and $0 \leq i_{1}, i_{2}, i_{3} \leq T-1$. Then

$$
o_{g, n, m}\left(a *_{g, m, p}^{n} b\right) w=o_{g, n, p}(a) o_{g, p, m}(b) w,
$$

where $w \in M(m)$. In particular,

$o_{g, n, m}\left(a *_{g, m}^{n} b\right) w=o_{g, n, m}(a) o_{g, m, m}(b) w, \quad o_{g, n, m}\left(a \bar{*}_{g, m}^{n} b\right) w=o_{g, n, n}(a) o_{g, n, m}(b) w$.

Proof. We first assume that $\overline{i_{1}-i_{2}} \neq s$. Then $o_{g, p, m}(b) w=0$. If $\overline{i_{2}-i_{3}}=r$, then $\overline{i_{1}-i_{3}} \neq \overline{r+s}$ and $o_{g, n, m}\left(a *_{g, m, p}^{n} b\right) w=0$. Otherwise, $\overline{i_{2}-i_{3}} \neq r$ and $a *_{g, m, p}^{n} b=0$. If $\overline{i_{2}-i_{3}} \neq r$, the proof is similar.

Finally we deal with the case that $\overline{i_{2}-i_{3}}=r, \overline{i_{1}-i_{2}}=s$. Let $\varepsilon=-1+\delta_{i_{1}}(r)+$ $\delta_{i_{3}}(T-r)$. Then on $M(m)$ we have

$$
\begin{aligned}
& o_{g, n, m}\left(a *_{g, m, p}^{n} b\right) \\
= & o_{g, n, m}\left(\sum_{i=0}^{l_{2}}(-1)^{i}\left(\begin{array}{c}
l_{1}+l_{3}-l_{2}+\varepsilon+i \\
i
\end{array}\right) \operatorname{Res}_{z} \frac{(1+z)^{\mathrm{wt} a-1+l_{1}+\delta_{i_{1}}(r)+r / T}}{z^{l_{1}+l_{3}-l_{2}+\varepsilon+i+1}} Y(a, z) b\right) \\
= & \sum_{i=0}^{l_{2}}(-1)^{i}\left(\begin{array}{c}
l_{1}+l_{3}-l_{2}+\varepsilon+i \\
i
\end{array}\right) \sum_{j=0}^{\mathrm{wt} a-1+l_{1}+\delta_{i_{1}}(r)+r / T}\left(\begin{array}{c}
\mathrm{wt} a-1+l_{1}+\delta_{i_{1}}(r)+r / T \\
j
\end{array}\right) \\
& \cdot\left(a_{j-l_{1}-l_{3}+l_{2}-\varepsilon-i-1} b\right)_{\mathrm{wt} a+\mathrm{wt} b-j+2 l_{1}-l_{2}+i+\varepsilon+\left(i_{1}-i_{3}\right) / T-1} \\
= & \sum_{i=0}^{l_{2}}(-1)^{i}\left(\begin{array}{c}
l_{1}+l_{3}-l_{2}+\varepsilon+i \\
i
\end{array}\right) \sum_{j=0}^{\mathrm{wt} a-1+l_{1}+\delta_{i_{1}}(r)+r / T}\left(\begin{array}{c}
\mathrm{wt} a-1+l_{1}+\delta_{i_{1}}(r)+r / T \\
j
\end{array}\right) \\
& \cdot \operatorname{Res}_{z_{2}} \operatorname{Res}_{z_{0}} z_{0}^{j-l_{1}-l_{3}+l_{2}-\varepsilon-i-1} z_{2}^{\mathrm{wt} a+\mathrm{wt} b-j+2 l_{1}-l_{2}+i+\varepsilon+\left(i_{1}-i_{3}\right) / T-1} Y_{M}\left(Y\left(a, z_{0}\right) b, z_{2}\right)
\end{aligned}
$$




$$
\begin{aligned}
& =\sum_{i=0}^{l_{2}}(-1)^{i}\left(\begin{array}{c}
l_{1}+l_{3}-l_{2}+\varepsilon+i \\
i
\end{array}\right) \operatorname{Res}_{z_{2}} \operatorname{Res}_{z_{0}} z_{0}^{-l_{1}-l_{3}+l_{2}-\varepsilon-i-1} \\
& \cdot\left(z_{2}+z_{0}\right)^{\mathrm{wt} a-1+l_{1}+\delta_{i_{1}}(r)+r / T} z_{2}^{\mathrm{wt} b+l_{1}-l_{2}+i-\delta_{i_{1}}(r)-r / T+\left(i_{1}-i_{3}\right) / T+\varepsilon} Y_{M}\left(Y\left(a, z_{0}\right) b, z_{2}\right) \\
& =\sum_{i=0}^{l_{2}}(-1)^{i}\left(\begin{array}{c}
l_{1}+l_{3}-l_{2}+\varepsilon+i \\
i
\end{array}\right) \sum_{j=0}^{\infty}\left(\begin{array}{c}
-l_{1}-l_{3}+l_{2}-\varepsilon-i-1 \\
j
\end{array}\right) \operatorname{Res}_{z_{1}} \operatorname{Res}_{z_{2}}\left(-z_{2}\right)^{j} \\
& \cdot z_{1}^{\mathrm{wt} a-1+l_{1}+\delta_{i_{1}}(r)+r / T} z_{1}^{-l_{1}-l_{3}+l_{2}-\varepsilon-i-1-j} \\
& \cdot z_{2}^{\mathrm{wt} b+l_{1}-l_{2}+i-\delta_{i_{1}}(r)-r / T+\left(i_{1}-i_{3}\right) / T+\varepsilon} Y_{M}\left(a, z_{1}\right) Y_{M}\left(b, z_{2}\right) \\
& -\sum_{i=0}^{l_{2}}(-1)^{i}\left(\begin{array}{c}
l_{1}+l_{3}-l_{2}+\varepsilon+i \\
i
\end{array}\right) \sum_{j=0}^{\infty}\left(\begin{array}{c}
-l_{1}-l_{3}+l_{2}-\varepsilon-i-1 \\
j
\end{array}\right) \\
& \operatorname{Res}_{z_{2}} \operatorname{Res}_{z_{1}} z_{1}^{j} z_{1}^{\mathrm{wt} a-1+l_{1}+\delta_{i_{1}}(r)+r / T}\left(-z_{2}\right)^{-l_{1}-l_{3}+l_{2}-\varepsilon-i-1-j} \\
& \cdot z_{2}^{\mathrm{wt} b+l_{1}-l_{2}+i-\delta_{i_{1}}(r)-r / T+\left(i_{1}-i_{3}\right) / T+\varepsilon} Y_{M}\left(b, z_{2}\right) Y_{M}\left(a, z_{1}\right) \\
& =\sum_{i=0}^{l_{2}}(-1)^{i}\left(\begin{array}{c}
l_{1}+l_{3}-l_{2}+\varepsilon+i \\
i
\end{array}\right) \sum_{j=0}^{\infty}\left(\begin{array}{c}
-l_{1}-l_{3}+l_{2}-\varepsilon-i-1 \\
j
\end{array}\right)(-1)^{j} \\
& \text { - } a_{\mathrm{wt} a-2+l_{2}-l_{3}-i-j+\delta_{i_{1}}(r)+r / T-\varepsilon} b_{\mathrm{wt} b+i+j+l_{1}-l_{2}-\delta_{i_{1}}(r)-r / T+\left(i_{1}-i_{3}\right) / T+\varepsilon} \\
& -\sum_{i=0}^{l_{2}}(-1)^{i}\left(\begin{array}{c}
l_{1}+l_{3}-l_{2}+\varepsilon+i \\
i
\end{array}\right) \sum_{j=0}^{\infty}\left(\begin{array}{c}
-l_{1}-l_{3}+l_{2}-q-i-1 \\
j
\end{array}\right) \\
& \cdot(-1)^{-l_{1}-l_{3}+l_{2}-q-i-1-j} b_{\mathrm{wt} b-l_{3}-j-1-\delta_{i_{1}}(r)-r / T+\left(i_{1}-i_{3}\right) / T} a_{\mathrm{wt} a-1+l_{1}+\delta_{i_{1}}(r)+r / T+j} \\
& =a_{\mathrm{wt} a-1+l_{2}-l_{3}+i_{2} / T-i_{3} / T} b_{\mathrm{wt} b-1+l_{1}-l_{2}+i_{1} / T-i_{2} / T} .
\end{aligned}
$$

The proof is complete.

Lemma 5.2. Let $m=l_{1}+i_{1} / T, n=l_{3}+i_{3} / T$ such that $l_{1}, l_{3} \in \mathbb{Z}_{+}, 0 \leq i_{1}, i_{3} \leq$ $T-1$. Then

$$
o_{g, n, m}(a)=0
$$

on $M(m)$, for all $a \in O_{g, n, m}(V)$.

Proof. Let $u \in V^{r}, v \in V^{s}$. We first prove that $o_{g, n, m}\left(u \circ_{g, m}^{n} v\right)=0$. This is clear if $\overline{i_{1}-i_{3}} \neq \overline{r+s}$. So we assume that $\overline{i_{1}-i_{3}}=\overline{r+s}$. Then

$$
\begin{aligned}
& o_{g, n, m}\left(u \circ_{g, m}^{n} v\right) \\
= & o_{g, n, m}\left(\operatorname{Res}_{z} \frac{(1+z)^{\mathrm{wt} u-1+\delta_{i_{1}}(r)+l_{1}+r / T}}{z^{l_{1}+l_{3}+\delta_{i_{1}}(r)+\delta_{i_{3}}(T-r)+1}} Y(u, z) v\right) \\
= & o_{g, n, m}\left(\sum_{j=0}^{\infty}\left(\begin{array}{c}
\mathrm{wt} u-1+\delta_{i_{1}}(r)+l_{1}+r / T \\
j
\end{array}\right) u_{j-l_{1}-l_{3}-\delta_{i_{1}}(r)-\delta_{i_{3}}(T-r)-1} v\right) \\
= & \sum_{j=0}^{\infty}\left(\begin{array}{c}
\mathrm{wt} u-1+\delta_{i_{1}}(r)+l_{1}+r / T \\
j
\end{array}\right) \\
& \cdot\left(u_{j-l_{1}-l_{3}-\delta_{i_{1}}(r)-\delta_{i_{3}}(T-r)-1} v\right)_{\mathrm{wt} t u+\mathrm{wt} t-1-j+2 l_{1}+\delta_{i_{1}}(r)+\delta_{i_{3}}(T-r)+\left(i_{1}-i_{3}\right) / T}
\end{aligned}
$$




$$
\begin{aligned}
& =\operatorname{Res}_{z_{2}} \operatorname{Res}_{z_{0}} \frac{\left(z_{2}+z_{0}\right)^{\mathrm{wt} u-1+\delta_{i_{1}}(r)+l_{1}+r / T} z_{2}^{\mathrm{wt} v+l_{1}+\delta_{i_{3}}(T-r)-r / T+\left(i_{1}-i_{3}\right) / T}}{z_{0}^{l_{1}+l_{3}+\delta_{i_{1}}(r)+\delta_{i_{3}}(T-r)+1}} \\
& \cdot Y_{M}\left(Y\left(u, z_{0}\right), z_{2}\right) v \\
& =\sum_{j=0}^{\infty}\left(\begin{array}{c}
-l_{1}-l_{3}-\delta_{i_{1}}(r)-\delta_{i_{3}}(T-r)-1 \\
j
\end{array}\right)(-1)^{j} \\
& \cdot \operatorname{Res}_{z_{1}} \operatorname{Res}_{z_{2}} \frac{z_{1}^{\mathrm{wt} u-1+\delta_{i_{1}}(r)+l_{1}+r / T}}{z_{1}^{l_{1}+l_{3}+\delta_{i_{1}}(r)+\delta_{i_{3}}(T-r)+1+j}} \\
& \cdot z_{2}^{\mathrm{wt} v+l_{1}+\delta_{i_{3}}(T-r)-r / T+\left(i_{1}-i_{3}\right) / T+j} Y_{M}\left(u, z_{1}\right) Y_{M}\left(v, z_{2}\right) \\
& -\sum_{j=0}^{\infty}\left(\begin{array}{c}
-l_{1}-l_{3}-\delta_{i_{1}}(r)-\delta_{i_{3}}(T-r)-1 \\
j
\end{array}\right) \\
& \cdot \operatorname{Res}_{z_{2}} \operatorname{Res}_{z_{1}} \frac{z_{2}^{\mathrm{wt} v+l_{1}+\delta_{i_{3}}(T-r)-r / T+\left(i_{1}-i_{3}\right) / T}}{z_{2}^{l_{1}+l_{3}+\delta_{i_{1}}(r)+\delta_{i_{3}}(T-r)+1+j}} \\
& \cdot(-1)^{l_{1}+l_{3}+\delta_{i_{1}}(r)+\delta_{i_{3}}(T-r)+1+j} z_{1}^{\mathrm{wt} u-1+\delta_{i_{1}}(r)+l_{1}+r / T+j} Y_{M}\left(v, z_{2}\right) Y_{M}\left(u, z_{1}\right) \\
& =\sum_{j=0}^{\infty}\left(\begin{array}{c}
-l_{1}-l_{3}-\delta_{i_{1}}(r)-\delta_{i_{3}}(T-r)-1 \\
j
\end{array}\right)(-1)^{j} \\
& \cdot u_{\mathrm{wt} u-2+r / T-l_{3}-\delta_{i_{3}}(T-r)-j} v_{\mathrm{wt} v+l_{1}+\delta_{i_{3}}(T-r)-r / T+\left(i_{1}-i_{3}\right) / T+j} \\
& -\sum_{j=0}^{\infty}\left(\begin{array}{c}
-l_{1}-l_{3}-\delta_{i_{1}}(r)-\delta_{i_{3}}(T-r)-1 \\
j
\end{array}\right)(-1)^{l_{1}+l_{3}+\delta_{i_{1}}(r)+\delta_{i_{3}}(T-r)+1+j} \\
& \cdot v_{\mathrm{wt} v}-r / T+\left(i_{1}-i_{3}\right) / T-l_{3}-\delta_{i_{1}}(r)-1-j u_{\mathrm{wt} u-1+\delta_{i_{1}}}(r)+l_{1}+r / T+j \\
& =0
\end{aligned}
$$

By Lemma 5.1 and the definition of $O_{g, n, m}(V)$, we know that $o_{g, n, m}(a)=0$, for all $a \in O_{g, n, m}^{\prime \prime}(V)+O_{g, n, m}^{\prime \prime \prime}(V)$.

Let $M=\bigoplus_{m \in \frac{1}{T} \mathbb{Z}_{+}} M(m)$ be an admissible $g$-twisted $V$-module with $M(0) \neq 0$. Then $\operatorname{Hom}_{\mathbb{C}}(M(m), M(n))$ is an $A_{g, n}(V)-A_{g, m}(V)$-bimodule such that $(a \cdot f \cdot b)(w)=$ $a f(b w)$ for $a \in A_{g, n}(V), b \in A_{g, m}(V), f \in \operatorname{Hom}_{\mathbb{C}}(M(m), M(n))$ and $w \in M(m)$. Set

$$
o_{g, n, m}(V)=\left\{o_{g, n, m}(v) \mid v \in V\right\} .
$$

By Lemmas 5.1 and 5.2 , we immediately have

Proposition 5.3. $o_{g, n, m}(V)$ is an $A_{g, n}(V)-A_{g, m}(V)$-subbimodule of $\operatorname{Hom}_{\mathbb{C}}(M(m)$, $M(n))$ and $v \mapsto o_{g, n, m}(v)$ for $v \in V$ induces an $A_{g, n}(V)-A_{g, m}(V)$-bimodule epimorphism from $A_{g, n, m}(V)$ to $o_{g, n, m}(V)$.

Proposition 5.4. For any $n \in \frac{1}{T} \mathbb{Z}_{+}, A_{g, n}(V)$ and $A_{g, n, n}(V)$ are the same.

Proof. The proof is similar to that of Proposition 4.6 of [DJ1].

Next we reconstruct the Verma type admissible $g$-twisted $V$-module $\bar{M}(U)$ generated by an $A_{g, m}(V)$-module $U$ by using the bimodules $A_{g, n, m}(V)$. Note that we do not assume that $U$ cannot factor through $A_{g, m-1 / T}(V)$ at this point. 
Set

$$
M(U)=\bigoplus_{n \in \frac{1}{T} \mathbb{Z}_{+}} A_{g, n, m}(V) \otimes_{A_{g, m}(V)} U
$$

Then $M(U)$ is $\frac{1}{T} \mathbb{Z}_{+}$-graded such that $M(U)(n)=A_{g, n, m}(V) \otimes_{A_{g, m}(V)} U$. For $u \in$ $V^{r}, p, n \in \frac{1}{T} \mathbb{Z}$, define an operator $u_{p}$ from $M(U)(n)$ to $M(U)(n+$ wt $u-p-1)$ by

$$
u_{p}(v \otimes w)=\left\{\begin{aligned}
\left(u *_{g, m, n}^{\mathrm{wt} u-p-1+n} v\right) \otimes w, & \text { if wt } u-1-p+n \geq 0, \\
0, & \text { if wt } u-1-p+n<0,
\end{aligned}\right.
$$

for $v \in A_{g, n, m}(V)$ and $w \in U$. It is clear that if $p \notin \mathbb{Z}+r / T$, then $u_{p}=0$.

Lemma 5.5. The action $u_{p}$ is well defined.

Proof. Let $v \in O_{g, n, m}(V)$ and $w \in U$. By Lemma 3.8, we have $u *_{g, m, n}^{\mathrm{wt} u-p-1+n} v \in$ $V *_{g, m, n}^{\mathrm{wt} u-p-1+n} O_{g, n, m}(V) \subseteq O_{g, \mathrm{wt} u-p-1+n, m}(V)$, so we have $u_{p}(v \otimes w)=0$. Now let $a \in A_{g, m}(V), v \in A_{g, n, m}(V), w \in U$. Then

$$
\begin{aligned}
& u_{p}\left(\left(v *_{g, m}^{n} a\right) \otimes w\right)=\left(u *_{g, m, n}^{\mathrm{wt} u-p-1+n}\left(v *_{g, m}^{n} a\right)\right) \otimes w \\
& \quad=\left(\left(u *_{g, m, n}^{\mathrm{wt} u-p-1+n} v\right) *_{g, m}^{\mathrm{wt} u-p-1+n} a\right) \otimes w \\
& \quad=\left(u *_{g, m, n}^{\mathrm{w} t u-p-1+n} v\right) \otimes a \cdot w=u_{p}(v \otimes a \cdot w) .
\end{aligned}
$$

Thus $u_{p}$ is well defined.

For short we set $M=M(U)$. Also let

$$
Y_{M}(u, z)=\sum_{p \in \mathbb{Z}+r / T} u_{p} z^{-p-1},
$$

for $u \in V^{r}$. It is our desire to prove that $\left(M(U), Y_{M}\right)$ is an admissible $g$-twisted $V$-module isomorphic to the $\bar{M}(U)$ given in Theorem 2.5 .

Lemma 5.6. For homogeneous $u \in V^{r}, v \otimes w \in A_{g, n, m}(V) \otimes_{A_{g, m}(V)} U$ and $p \in$ $\mathbb{Z}+r / T$, we have

(1) $u_{p}(v \otimes w)=0$, for $p$ sufficiently large;

(2) $Y_{M}(\mathbf{1}, z)=\operatorname{id}_{M}$.

Proof. From the definition, (1) is obvious. We now prove (2). By the definition of $u_{p}$, we have

$$
\begin{aligned}
& \mathbf{1}_{p}(v \otimes w)=\left(1 *_{g, m, n}^{-p-1+n} v\right) \otimes w \\
& =\sum_{i=0}^{l_{2}}(-1)^{i}\left(\begin{array}{c}
l_{1}-p-1+i \\
i
\end{array}\right) \operatorname{Res}_{z} \frac{(1+z)^{l_{1}}}{z^{l_{1}-p+i}} Y(1, z) v \otimes w,
\end{aligned}
$$

where $p \in \mathbb{Z}, m=l_{1}+i_{1} / T, n=l_{2}+i_{2} / T$ such that $l_{1}, l_{2} \in \mathbb{Z}_{+}$and $0 \leq i_{1}, i_{2}<T$. Thus $\mathbf{1}_{p}(v \otimes w)=0$ if $p<-1$ and $\mathbf{1}_{-1}(v \otimes w)=v \otimes w$. By the definition of $\mathbf{1}_{p}$, $\mathbf{1}_{p}(v \otimes w)=0$ if $p \geq l_{2}$. If $-1<p<l_{2}$, then

$$
\begin{aligned}
& \mathbf{1}_{p}(v \otimes w)=\sum_{i=0}^{p+1}(-1)^{i}\left(\begin{array}{c}
l_{1}-p-1+i \\
i
\end{array}\right)\left(\begin{array}{c}
l_{1} \\
p+1-i
\end{array}\right) v \otimes w \\
& =\sum_{i=0}^{p+1}(-1)^{i+p+1}\left(\begin{array}{c}
l_{1} \\
p+1
\end{array}\right)\left(\begin{array}{c}
p+1 \\
i
\end{array}\right) v \otimes w \\
& =0 .
\end{aligned}
$$

As a result we have $Y_{M}(\mathbf{1}, z)=\operatorname{id}_{M}$. 
The main axiom in the definition of admissible $g$-twisted $V$-modules is the twisted Jacobi identity. As we have already mentioned in Section 2, the twisted Jacobi identity is equivalent to the commutator formula (2.3) and the associativity (2.2). We have the commutator formula:

Lemma 5.7. For $a \in V^{r}, b \in V^{s}$, we have

$$
\left[Y_{M}\left(a, z_{1}\right), Y_{M}\left(b, z_{2}\right)\right]=\operatorname{Res}_{z_{0}} z_{2}^{-1}\left(\frac{z_{1}-z_{0}}{z_{2}}\right)^{-r / T} \delta\left(\frac{z_{1}-z_{0}}{z_{2}}\right) Y_{M}\left(Y\left(a, z_{0}\right) b, z_{2}\right),
$$

or equivalently, for $p \in \mathbb{Z}+r / T, q \in \mathbb{Z}+s / T$,

$$
\left[a_{p}, b_{q}\right]=\sum_{i=0}^{\infty}\left(\begin{array}{l}
p \\
i
\end{array}\right)\left(a_{i} b\right)_{p+q-i} .
$$

Proof. We need to prove that

$$
\left(a_{p} b_{q}-b_{q} a_{p}\right)(v \otimes w)=\sum_{i=0}^{\infty}\left(\begin{array}{c}
p \\
i
\end{array}\right)\left(a_{i} b\right)_{p+q-i}(v \otimes w)
$$

for $p \in \mathbb{Z}+r / T, q \in \mathbb{Z}+s / T$ and $v \otimes w \in A_{g, n, m}(V) \otimes_{A_{g, m}(V)} U$. This is clear from the definition of the actions if wt $a+\mathrm{wt} b-p-q-2+n<0$. We now assume that $\mathrm{wt} a+\mathrm{wt} b-p-q-2+n \geq 0$.

If wt $a-p-1+n \geq 0$, wt $b-q-1+n \geq 0$, then by Lemma 3.4 we have

$$
\begin{aligned}
& a_{p} b_{q}(v \otimes w)-b_{q} a_{p}(v \otimes w) \\
= & a_{p}\left(b *_{g, m, n}^{\mathrm{wt} b-q-1+n} v\right) \otimes w-b_{q}\left(a *_{g, m, n}^{\mathrm{wt} a-p-1+n} v\right) \otimes w \\
= & \left(a *_{g, m, \mathrm{wt} b-q-1+n}^{\mathrm{wt} a+\mathrm{wt} b-p-q-2+n}\left(b *_{g, m, n}^{\mathrm{wt} b-q-1+n} v\right)\right) \otimes w \\
& -\left(b *_{g, m, \mathrm{wt} a-p-1+n}^{\mathrm{wt} a+\mathrm{wt} b-p-q-2+n}\left(a *_{g, m, n}^{\mathrm{wt} a-p-1+n} v\right)\right) \otimes w \\
= & \left(\left(a *_{g, n, \mathrm{wt} b-q-1+n}^{\mathrm{wt} a+\mathrm{wt} b-p-q-2+n} b\right) *_{g, m, n}^{\mathrm{wt} a+\mathrm{wt} b-p-q-2+n} v\right) \otimes w \\
& -\left(\left(b *_{g, n, \mathrm{wt} a-p-1+n}^{\mathrm{wt} a+\mathrm{wt} b-p-q-2+n} a\right) *_{g, m, n}^{\mathrm{wt} a+w b-p-q-2+n} v\right) \otimes w \\
= & \left(\left(\operatorname{Res}_{z}(1+z)^{p} Y_{M}(a, z) b\right) * *_{g, m, n}^{\mathrm{wt} a+\mathrm{wt} b-p-q-2+n} v\right) \otimes w \\
= & \left(\sum_{i=0}^{\infty}\left(\begin{array}{l}
p \\
i
\end{array}\right)\left(a_{i} b\right) *{ }_{g, m, n}^{\mathrm{wt} a+\mathrm{wt} b-p-q-2+n} v\right) \otimes w \\
= & \sum_{i=0}^{\infty}\left(\begin{array}{l}
p \\
i
\end{array}\right)\left(a_{i} b\right)_{p+q-i}(v \otimes w) .
\end{aligned}
$$

If wt $a-p-1+n<0$, wt $b-q-1+n \geq 0$, then $b_{q} a_{p}(v \otimes w)=0$ and

$$
\begin{aligned}
& a_{p} b_{q}(v \otimes w)-b_{q} a_{p}(v \otimes w)=\left(\left(a *_{g, n, \mathrm{wt} b-q-1+n}^{\mathrm{wt} a+\mathrm{wt} b-p-q-2+n} b\right) *{ }_{g, m, n}^{\mathrm{wt} p+\mathrm{wt} b-p-q-2+n} v\right) \otimes w \\
& \quad=\left(\left(\operatorname{Res}_{z}(1+z)^{p} Y_{M}(a, z) b\right) *_{g, m, n}^{\mathrm{wt} a+\mathrm{wt} b-p-q-2+n} v\right) \otimes w \\
& \quad=\sum_{i=0}^{\infty}\left(\begin{array}{l}
p \\
i
\end{array}\right)\left(a_{i} b\right)_{p+q-i}(v \otimes w)
\end{aligned}
$$

where we have used Lemma 5.8 below. If wta $-p-1+n \geq 0$, wt $b-q-1+n<0$, the proof is similar. 
Lemma 5.8. Let $u \in V^{r}, v \in V^{s}$ and $m=l_{1}+i_{1} / T, n=l_{3}+i_{3} / T, p=l_{2}+i_{2} / T$ such that $l_{1}, l_{3} \in \mathbb{Z}_{+}, l_{2} \in \mathbb{Z}, 0 \leq i_{1}, i_{2}, i_{3}<T$, and $\overline{i_{2}-i_{3}}=r, \overline{i_{1}-i_{2}}=s$. If $p \geq 0, m+n-p<0$, then

$$
u *_{g, m, p}^{n} v-\operatorname{Res}_{z}(1+z)^{\mathrm{wt} u-1+p-n} Y(u, z) v \in O_{g, n, m}^{\prime}(V)
$$

and if $p<0, m+n-p \geq 0$, then

$$
-v *_{g, m, m+n-p}^{n} u-\operatorname{Res}_{z}(1+z)^{\mathrm{wt} u-1+p-n} Y(u, z) v \in O_{g, n, m}^{\prime}(V) .
$$

Proof. We first assume that $p \geq 0, m+n-p<0$. By (3.1), $-l_{1}-l_{3}+l_{2}-\varepsilon-1 \in \mathbb{Z}_{+}$. Using the definition we have

$$
\begin{aligned}
u *_{g, m, p}^{n} v= & \sum_{i=0}^{l_{2}}(-1)^{i}\left(\begin{array}{c}
l_{1}+l_{3}-l_{2}+\varepsilon+i \\
i
\end{array}\right) \\
& \cdot \operatorname{Res}_{z} \frac{(1+z)^{\mathrm{wt} u-1+l_{1}+\delta_{i_{1}}(r)+r / T}}{z^{l_{1}+l_{3}-l_{2}+\varepsilon+i+1}} Y(u, z) v .
\end{aligned}
$$

Since $\operatorname{Res}_{z} Y(u, z) v \frac{(1+z)^{\mathrm{wt} u-1+l_{1}+\delta_{i_{1}}(r)+r / T}}{z^{l_{1}+l_{3}-l_{2}+\varepsilon+i+1}} \in O_{g, n, m}^{\prime}(V)$ if $i>l_{2}$, by Lemma 3.3 we see that

$$
\begin{aligned}
& u *_{g, m, p}^{n} v \equiv \sum_{i=0}^{\infty}\left(\begin{array}{c}
-l_{1}-l_{3}+l_{2}-\varepsilon-1 \\
i
\end{array}\right) \operatorname{Res}_{z} Y(u, z) v \frac{(1+z)^{\mathrm{wt} u-1+l_{1}+\delta_{i_{1}}(r)+r / T}}{z^{l_{1}+l_{3}-l_{2}+\varepsilon+i+1}} \\
& =\operatorname{Res}_{z}(1+z)^{\mathrm{wt} u-1+p-n} Y(u, z) v
\end{aligned}
$$

where in the last step we have used the fact that $\delta_{i_{3}}(T-r)=\left(r+i_{3}-i_{2}\right) / T$. So in this case we are done.

If $p<0, m+n-p \geq 0$, then the result in the first case gives

$$
v *_{g, m, m+n-p}^{n} u \equiv \operatorname{Res}_{z}(1+z)^{\mathrm{wt} v-1+m-p} Y(v, z) u
$$

modulo $O_{g, n, m}^{\prime}(V)$. Using the identity

$$
Y(v, z) u \equiv(1+z)^{-\mathrm{wt} u-\mathrm{wt} v-m+n} Y\left(u, \frac{-z}{1+z}\right) v
$$

modulo $O_{g, n, m}^{\prime}(V)$ we see that

$$
\begin{aligned}
& \operatorname{Res}_{z}(1+z)^{\mathrm{wt} v-1+m-p} Y(v, z) u \equiv \operatorname{Res}_{z}(1+z)^{-\operatorname{wt} u-1+n-p} Y\left(u, \frac{-z}{1+z}\right) v \\
& \quad=-\operatorname{Res}_{z}(1+z)^{\mathrm{wt} u-1+p-n} Y(u, z) v .
\end{aligned}
$$

The proof is complete.

Lemma 5.9. Let $n=l_{3}+i_{3} / T \in \frac{1}{T} \mathbb{Z}_{+}$with $l_{3} \in \mathbb{Z}_{+}$and $0 \leq i_{3}<T$. Then for $a \in V^{r}$ and $i \in \mathbb{Z}_{+}$, we have

$$
\begin{aligned}
& \operatorname{Res}_{z_{0}} z_{0}^{i}\left(z_{0}+z_{2}\right)^{\mathrm{wt} a-1+l_{3}+\delta_{i_{3}}(r)+r / T} Y_{M}\left(a, z_{0}+z_{2}\right) Y_{M}\left(b, z_{2}\right) \\
= & \operatorname{Res}_{z_{0}} z_{0}^{i}\left(z_{2}+z_{0}\right)^{\mathrm{wt} a-1+l_{3}+\delta_{i_{3}}(r)+r / T} Y_{M}\left(Y\left(a, z_{0}\right) b, z_{2}\right)
\end{aligned}
$$

on $M(U)(n)$. 
Proof. Note that $a_{\mathrm{wt} a-1+l_{3}+\delta_{i_{3}}(r)+r / T+j}=0$ on $M(U)(n)$ for any nonnegative integer $j$. Then

$$
\operatorname{Res}_{z_{1}}\left(z_{1}-z_{2}\right)^{i} z_{1}^{\mathrm{wt} a-1+l_{3}+\delta_{i_{3}}(r)+r / T} Y_{M}\left(b, z_{2}\right) Y_{M}\left(a, z_{1}\right)=0
$$

on $M(U)(n)$ and

$$
\begin{aligned}
& \operatorname{Res}_{z_{0}} z_{0}^{i}\left(z_{0}+z_{2}\right)^{\mathrm{wt} a-1+l_{3}+\delta_{i_{3}}(r)+r / T} Y_{M}\left(a, z_{0}+z_{2}\right) Y_{M}\left(b, z_{2}\right) \\
&=\operatorname{Res}_{z_{1}}\left(z_{1}-z_{2}\right)^{i} z_{1}^{\mathrm{wt} a-1+l_{3}+\delta_{i_{3}}(r)+r / T}\left(Y_{M}\left(a, z_{1}\right) Y_{M}\left(b, z_{2}\right)-Y_{M}\left(b, z_{2}\right) Y_{M}\left(a, z_{1}\right)\right) \\
&=\operatorname{Res}_{z_{1}}\left(z_{1}-z_{2}\right)^{i} z_{1}^{\operatorname{wt} a-1+l_{3}+\delta_{i_{3}}(r)+r / T}\left[Y_{M}\left(a, z_{1}\right), Y_{M}\left(b, z_{2}\right)\right] \\
&=\operatorname{Res}_{z_{0}} \operatorname{Res}_{z_{1}}\left(z_{1}-z_{2}\right)^{i} z_{1}^{\mathrm{wt} a-1+l_{3}+\delta_{i_{3}}(r)+r / T} \\
& \cdot\left(\frac{z_{1}-z_{0}}{z_{2}}\right)^{-r / T} z_{2}^{-1} \delta\left(\frac{z_{1}-z_{0}}{z_{2}}\right) Y_{M}\left(Y\left(a, z_{0}\right) b, z_{2}\right) \\
&= \operatorname{Res}_{z_{0}} \operatorname{Res}_{z_{1}} z_{0}^{i}\left(z_{2}+z_{0}\right)^{\mathrm{wt} a-1+l_{3}+\delta_{i_{3}}(r)+r / T} \\
& \cdot\left(\frac{z_{1}-z_{0}}{z_{2}}\right)^{-r / T} z_{1}^{-1} \delta\left(\frac{z_{2}+z_{0}}{z_{1}}\right) Y_{M}\left(Y\left(a, z_{0}\right) b, z_{2}\right) \\
&= \operatorname{Res}_{z_{0}} z_{0}^{i}\left(z_{2}+z_{0}\right)^{\mathrm{wt} a-1+l_{3}+\delta_{i_{3}}(r)+r / T} Y_{M}\left(Y\left(a, z_{0}\right) b, z_{2}\right),
\end{aligned}
$$

where we have used Lemma 5.7 .

Lemma 5.10. Let $n=l_{3}+i_{3} / T \in \frac{1}{T} \mathbb{Z}_{+}$with $l_{3} \in \mathbb{Z}_{+}$and $0 \leq i_{3}<T$. Then for $a \in V^{r}$ and $l \in \mathbb{Z}_{+} \backslash\{0\}$, we have

$$
\begin{aligned}
& \operatorname{Res}_{z_{0}} z_{0}^{-l}\left(z_{2}+z_{0}\right)^{\mathrm{wt} a+q} z_{2}^{\mathrm{wt} b-q} Y_{M}\left(Y\left(a, z_{0}\right) b, z_{2}\right) \\
& =\operatorname{Res}_{z_{0}} z_{0}^{-l}\left(z_{0}+z_{2}\right)^{\mathrm{wt} a+q} z_{2}^{\mathrm{wt} b-q} Y_{M}\left(a, z_{0}+z_{2}\right) Y_{M}\left(b, z_{2}\right)
\end{aligned}
$$

on $M(U)(n)$, where $q=-1+l_{3}+\delta_{i_{3}}(r)+r / T$.

Proof. Assume that $b \in V^{s}$. Take $v \otimes w \in A_{g, n, m}(V) \otimes_{A_{g, m}(V)} U=M(U)(n)$. Then

$$
\begin{aligned}
& \operatorname{Res}_{z_{0}} z_{0}^{-l}\left(z_{2}+z_{0}\right)^{\mathrm{wt} a+q} z_{2}^{\mathrm{wt} b-q} Y_{M}\left(Y\left(a, z_{0}\right) b, z_{2}\right)(v \otimes w) \\
& =\sum_{j \in \mathbb{Z}_{+}}\left(\begin{array}{c}
\mathrm{wt} a+q \\
j
\end{array}\right) z_{2}^{\mathrm{wt} a+\mathrm{wt} b-j} Y_{M}\left(a_{j-l} b, z_{2}\right)(v \otimes w) \\
& =\sum_{j \in \mathbb{Z}_{+}}\left(\begin{array}{c}
\mathrm{wt} a+q \\
j
\end{array}\right) \sum_{k \in \mathbb{Z}_{+}+\overline{i_{3}-r-s} / T} z_{2}^{-l+k-n+1}\left(a_{j-l} b\right)_{\mathrm{wt} a+\mathrm{wt} b-j+l-2-k+n}(v \otimes w) \\
& =\sum_{k \in \mathbb{Z}_{+}+\overline{i_{3}-r-s} / T} z_{2}^{-l+k-n+1} \sum_{j \in \mathbb{Z}_{+}}\left(\begin{array}{c}
\mathrm{wt} a+q \\
j
\end{array}\right)\left(\left(a_{j-l} b\right) *_{g, m, n}^{k} v\right) \otimes w \\
& =\sum_{k \in \mathbb{Z}_{+}+\overline{i_{3}-r-s} / T} z_{2}^{-l+k-n+1}\left(\left(\operatorname{Res}_{z} \frac{(1+z)^{\mathrm{wt} a+q}}{z^{l}} Y(a, z) b\right) *_{g, m, n}^{k} v\right) \otimes w .
\end{aligned}
$$


On the other hand, we have

$$
\begin{aligned}
& \operatorname{Res}_{z_{0}} z_{0}^{-l}\left(z_{0}+z_{2}\right)^{\mathrm{wt} a+q} z_{2}^{\mathrm{wt} b-q} Y_{M}\left(a, z_{0}+z_{2}\right) Y_{M}\left(b, z_{2}\right)(v \otimes w) \\
& =\sum_{i \in \mathbb{Z}_{+}}\left(\begin{array}{c}
-l \\
i
\end{array}\right)(-1)^{i} a_{\mathrm{wt} a+q-l-i} z_{2}^{\mathrm{wt} b-q+i} Y_{M}\left(b, z_{2}\right)(v \otimes w) \\
& =\sum_{i \in \mathbb{Z}_{+}}\left(\begin{array}{c}
-l \\
i
\end{array}\right)(-1)^{i} a_{\mathrm{wt} a+q-l-i} \sum_{j \geq-n, j \in \mathbb{Z}-s / T} z_{2}^{-q+i+j} b_{\mathrm{wt} b-1-j}(v \otimes w) \\
& =\sum_{i \in \mathbb{Z}_{+}} \sum_{\substack{j \geq-n, j \in \mathbb{Z}-s / T \\
l+i+j \geq 1+q-n}}\left(\begin{array}{c}
-l \\
i
\end{array}\right)(-1)^{i} z_{2}^{-q+i+j}\left(a *_{g, m, j+n}^{l+i+j-1-q+n}\left(b *_{g, m, n}^{j+n} v\right)\right) \otimes w \\
& =\sum_{k \in \mathbb{Z}_{+}+\overline{i_{3}-r-s} / T} \sum_{\substack{j \in \mathbb{Z}-s / T \\
-n \leq j \leq k+1+q-n-l}} z_{2}^{-l+k-n+1}(-1)^{k+1+q-n-j-l} \\
& \cdot\left(\begin{array}{c}
-l \\
k+1+q-n-j-l
\end{array}\right)\left(\left(a *_{g, n, j+n}^{k} b\right) *_{g, m, n}^{k} v\right) \otimes w \\
& =\sum_{k \in \mathbb{Z}_{+}+\bar{i}_{3}-r-s / T} \sum_{\substack{j \in \mathbb{Z}+\left(i_{3}-s\right) / T \\
0 \leq j \leq k+1+q-l}} z_{2}^{-l+k-n+1}(-1)^{k+1+q-j-l}\left(\begin{array}{c}
-l \\
k+1+q-j-l
\end{array}\right) \\
& \cdot\left(\left(a *_{g, n, j}^{k} b\right) *_{g, m, n}^{k} v\right) \otimes w .
\end{aligned}
$$

So it is enough to prove that

$$
\sum_{\substack{j \in \mathbb{Z}+\left(i_{3}-s\right) / T \\
0 \leq j \leq k+1+q-l}}(-1)^{k+1+q-j-l}\left(\begin{array}{c}
-l \\
k+1+q-j-l
\end{array}\right) a *_{g, n, j}^{k} b=\operatorname{Res}_{z} \frac{(1+z)^{\mathrm{wt} a+q}}{z^{l}} Y(a, z) b,
$$

for $k \geq 0$. Let $j=p+\left(i_{3}-s\right) / T-\delta_{i_{3}}(s)+1, k=l_{1}+\left(i_{3}-r-s\right) / T$. Note that $q=-1+l_{3}+\delta_{i_{3}}(r)+r / T$, so

$$
\begin{aligned}
& \sum_{\substack{j \in \mathbb{Z}+\left(i_{3}-s\right) / T \\
0 \leq j \leq k+1+q-l}}(-1)^{k+1+q-j-l}\left(\begin{array}{c}
-l \\
k+1+q-j-l
\end{array}\right) a *_{g, n, j}^{k} b \\
& =\sum_{p=0}^{l_{1}+l_{3}+\delta_{i_{3}}(r)+\delta_{i_{3}}(s)-1-l}(-1)^{l_{1}+l_{3}+\delta_{i_{3}}(r)+\delta_{i_{3}}(s)-1-l-p} \\
& \cdot\left(\begin{array}{c}
-l \\
l_{1}+l_{3}+\delta_{i_{3}}(r)+\delta_{i_{3}}(s)-1-l-p
\end{array}\right) \\
& \cdot \sum_{i=0}^{p}(-1)^{i}\left(\begin{array}{c}
l_{1}+l_{3}+\delta_{i_{3}}(r)+\delta_{i_{3}}(s)-2-p+i \\
i
\end{array}\right) \\
& \cdot \operatorname{Res}_{z} \frac{(1+z)^{\mathrm{wt} a+q}}{z^{l_{1}+l_{3}+\delta_{i_{3}}(r)+\delta_{i_{3}}(s)-1-p+i}} Y(a, z) b \\
& =\sum_{i=0}^{l_{1}+l_{3}+\delta_{i_{3}}(r)+\delta_{i_{3}}(s)-1-l}(-1)^{p}\left(\begin{array}{c}
-l \\
p
\end{array}\right) \\
& \sum_{p=0}^{l_{1}+l_{3}+\delta_{i_{3}}(r)+\delta_{i_{3}}(s)-1-l-p}\left(\begin{array}{c}
l+p+i-1 \\
\operatorname{Res}_{z} \frac{(1+z)^{\mathrm{wt} a+q}}{z^{l+p+i}} Y(a, z) b .
\end{array}\right)
\end{aligned}
$$


By Proposition 5.3 in [DJ1, we have

$$
\begin{array}{r}
\sum_{p=0}^{l_{1}+l_{3}+\delta_{i_{3}}(r)+\delta_{i_{3}}(s)-1-l}(-1)^{p}\left(\begin{array}{c}
-l \\
p
\end{array}\right) \sum_{i=0}^{l_{1}+l_{3}+\delta_{i_{3}}(r)+\delta_{i_{3}}(s)-1-l-p}(-1)^{i}\left(\begin{array}{c}
l+p+i-1 \\
i
\end{array}\right) \frac{1}{z^{l+p+i}} \\
=\frac{1}{z^{l}} .
\end{array}
$$

This finishes the proof.

Corollary 5.11. Let $n=l_{3}+i_{3} / T \in \frac{1}{T} \mathbb{Z}_{+}$with $l_{3} \in \mathbb{Z}_{+}$and $0 \leq i_{3}<T$. Then for $a \in V^{r}$, we have

$$
\left(z_{2}+z_{0}\right)^{\mathrm{wt} a+q} Y_{M}\left(Y\left(a, z_{0}\right) b, z_{2}\right)=\left(z_{0}+z_{2}\right)^{\mathrm{wt} a+q} Y_{M}\left(a, z_{0}+z_{2}\right) Y_{M}\left(b, z_{2}\right)
$$

on $M(U)(n)$, where $q=-1+l_{3}+\delta_{i_{3}}(r)+r / T$.

Theorem 5.12. Let $U$ be an $A_{g, m}(V)$-module. Then $M(U)=\bigoplus_{n \in \frac{1}{T} \mathbb{Z}_{+}} A_{g, n, m}(V)$ $\otimes_{A_{g, m}(V)} U$ is an admissible $g$-twisted $V$-module with $M(U)(n)=A_{g, n, m}(V) \otimes_{A_{g, m}(V)}$ $U$ and with the following universal property: for any weak $g$-twisted $V$-module $W$ and any $A_{g, m}(V)$-morphism $\sigma: U \rightarrow \Omega_{m}(W)$, there is a unique homomorphism $\bar{\sigma}: M(U) \rightarrow W$ of weak $g$-twisted $V$-modules that extends $\sigma$. Moreover, if $U$ cannot factor through $A_{g, m-1 / T}(V)$, then $M(U)(0) \neq 0$.

It is clear from Theorem 5.12 that $M(U)$ is isomorphic to the $\bar{M}(U)$ given in Theorem 2.5. We call $M(U)$ the Verma type admissible $g$-twisted $V$-module generated by an $A_{g, m}(V)$-module $U$.

\section{6. $g$-RATIONALITY}

We use the bimodule theory developed in the previous sections to prove another main theorem in this paper. That is, $V$ is $g$-rational if and only if $A_{g}(V)$ is semisimple and any irreducible admissible $g$-twisted $V$-module is ordinary. In the case $g=1$, this result has been obtained previously in DJ2] and DJ3.

We need several lemmas. Let $A$ be an associative algebra and $U$ a left $A$-module. It is well known that the linear dual $U^{*}=\operatorname{Hom}_{\mathbb{C}}(U, \mathbb{C})$ is naturally a right $A$-module such that $(f a)(u)=f(a u)$ for $a \in A, f \in U^{*}$ and $u \in U$.

Lemma 6.1. Let $V$ be a vertex operator algebra. Assume that $A_{g}(V)$ is semisimple and $U^{i}$ for $i=1, \cdots, s$ are all the inequivalent irreducible $A_{g}(V)$-modules. Let $M\left(U^{i}\right)=\bigoplus_{n \in \frac{1}{T} \mathbb{Z}_{+}} M\left(U^{i}\right)(n)$ be the Verma type admissible $g$-twisted $V$-module generated by the $A_{g}(V)$-module $U^{i}$. Then as an $A_{g, n}(V)-A_{g}(V)$-bimodule,

$$
A_{g, n, 0}(V) \cong \bigoplus_{i=1}^{s} M\left(U^{i}\right)(n) \otimes\left(U^{i}\right)^{*} .
$$

Proof. The proof is similar to that of Lemma 3.1 in DJ3].

Lemma 6.2. Let $V$ be a simple vertex operator algebra such that $A_{g}(V)$ is finite dimensional. Then there exists $N \in \frac{1}{T} \mathbb{Z}_{+}$such that for any irreducible $A_{g}(V)$-module $U$ and the irreducible admissible g-twisted $V$-module $L(U)=\sum_{m \in \frac{1}{T} \mathbb{Z}_{+}} L(U)(m)$ generated by $U, L(U)(n) \neq 0$, for all $n \in \frac{1}{T} \mathbb{Z}_{+}, n>N$. 
Proof. Since $A_{g}(V)$ is finite dimensional, there are only finitely many irreducible admissible $g$-twisted $V$-modules. So it suffices to prove that for the irreducible admissible $g$-twisted $V$-module $W=\bigoplus_{m \in \frac{1}{T} \mathbb{Z}_{+}} W(m)$ there exists $N \in \frac{1}{T} \mathbb{Z}_{+}$such that $W(n) \neq 0$ for all $n \in \frac{1}{T} \mathbb{Z}_{+}, n>N$.

For $i=0, \cdots, T-1$ we set $W^{i}=\bigoplus_{m \in \mathbb{Z}_{+}} W\left(m+\frac{i}{T}\right)$. Note from [DM] and DLM0] that the $g$-invariant $V^{\langle g\rangle}$ again is a simple vertex operator algebra. From the definition of admissible $g$-twisted $V$-modules we see that each $W^{i}$ is an irreducible admissible $V^{\langle g\rangle}$-module (see [DY]). It is clear that for each $i$ there exists $n_{i} \geq 0$ such that $W^{i}(s) \neq 0$ if $s>n_{i}$ and $s \in \frac{i}{T}+\mathbb{Z}_{+}$. Take $N$ to be the maximum of $n_{i}$ for $i=0, \cdots, T-1$ and the lemma follows.

For $m, n, p \in \frac{1}{T} \mathbb{Z}_{+}$, let

$$
A_{g, n, p}(V) *_{g, m, p}^{n} A_{g, p, m}(V)=\left\{a *_{g, m, p}^{n} b \mid a \in A_{g, n, p}(V), b \in A_{g, p, m}(V)\right\} .
$$

Then $A_{g, n, p}(V) *_{g, m, p}^{n} A_{g, p, m}(V)$ is an $A_{g, n}(V)-A_{g, m}(V)$-subbimodule of $A_{g, n, m}(V)$ by Proposition 4.4

Lemma 6.3. Let $V$ be a simple vertex operator algebra such that $A_{g}(V)$ is semisimple. Then there exists $N \in \frac{1}{T} \mathbb{Z}_{+}$such that

$$
A_{g, 0, m}(V) *_{g, 0, m}^{0} A_{g, m, 0}(V)=A_{g}(V)
$$

for all $m \in \frac{1}{T} \mathbb{Z}_{+}, m>N$.

Proof. Let $N$ be the same as in Lemma 6.2. For any $n \in \frac{1}{T} \mathbb{Z}_{+}$, it is easy to see that $A_{g, 0, n}(V) *_{g, 0, n}^{0} A_{g, n, 0}(V)$ is a two-sided ideal of $A_{g}(V)$. Let $U$ be an irreducible module of $A_{g}(V)$ and suppose that for some $m \in \frac{1}{T} \mathbb{Z}_{+}, m>N$,

$$
A_{g, 0, m}(V) *_{g, 0, m}^{0} A_{g, m, 0}(V) \otimes U=0 .
$$

Let $M(U)$ be the Verma type admissible $g$-twisted $V$-module generated by $U$ and $M^{\prime}(U)$ the maximal proper admissible $g$-twisted submodule of $M(U)$. Similar to the proof of Proposition 4.5.6 of [LL] (see also $[\overline{D M}]$ ), we have that

$$
M^{\prime \prime}(U)=\operatorname{span}\left\{u_{p} w \mid u \in V, p \in \mathbb{Q}, w \in M(U)(m)\right\}
$$

is an admissible $g$-twisted $V$-submodule of $M(U)$ generated by $M(U)(m)$. By (6.1) we know that $M^{\prime \prime}(U)(0)=0$. So $M^{\prime \prime}(U)$ is a proper admissible $g$-twisted $V$-submodule of $M(U)$ and $M^{\prime \prime}(U) \subset M^{\prime}(U)$.

Let $W(U)=M(U) / M^{\prime}(U)$. Then $W(U)$ is the irreducible admissible $g$-twisted $V$-module generated by $U$ and $W(U)(m)=0$. This is in contradiction with Lemma 6.2. Thus for all $m \in \frac{1}{T} \mathbb{Z}_{+}, m>N$,

$$
A_{g, 0, m}(V) *_{g, 0, m}^{0} A_{g, m, 0}(V) \otimes U \cong U .
$$

Now the lemma follows from Lemma 6.1.

Recall from Proposition 4.4 that $\varphi: A_{g, n, p}(V) \otimes_{A_{g, p}(V)} A_{g, p, m}(V) \rightarrow A_{g, n, m}(V)$ is an $A_{g, n}(V)-A_{g, m}(V)$-bimodule homomorphism defined by

$$
\varphi(u \otimes v)=u *_{g, m, p}^{n} v,
$$

for $u \in A_{g, n, p}(V), v \in A_{g, p, m}(V)$ and $m, p, n \in \frac{1}{T} \mathbb{Z}_{+}$. The following lemma is an immediate consequence of Lemma 6.3. 
Lemma 6.4. Let $V$ be a simple vertex operator algebra such that $A_{g}(V)$ is semisimple. Then there exists $N \in \frac{1}{T} \mathbb{Z}_{+}$such that the $A_{g}(V)-A_{g}(V)$-bimodule homomorphism $\varphi$ from $A_{g, 0, n}(V) \otimes_{A_{g, n}(V)} A_{g, n, 0}(V)$ to $A_{g}(V)$ is an isomorphism for each $n \in \frac{1}{T} \mathbb{Z}_{+}, n>N$.

Theorem 6.5. Let $V$ be a simple vertex operator algebra such that $A_{g}(V)$ is semisimple. Let $U$ be an irreducible module of $A_{g}(V)$. Then the Verma type admissible g-twisted $V$-module $M(U)=\bigoplus_{n \in \frac{1}{T} \mathbb{Z}_{+}} A_{g, n, 0}(V) \otimes_{A_{g}(V)} U$ generated by $U$ is irreducible.

Proof. The same proof of Theorem 3.4 of DJ3 is valid here.

We have already mentioned that the Verma type admissible $g$-twisted $V$-module $M(U)$ generated by an irreducible $A_{g}(V)$-module $U$ in general is not irreducible. The assumption that $A_{g}(V)$ is semisimple is crucial. This result is a foundation of Theorem 6.7 below.

As in DJ3 we now introduce an invariant bilinear pairing $(\cdot, \cdot)$ on $M\left(U^{*}\right) \times$ $M(U)$, for an $A_{g, m}(V)$-module $U$, which is an analogue of the contravariant forms for Kac-Moody Lie algebras and the Virasoro algebra. This bilinear pairing will also be helpful to the proof of Theorem 6.7 .

Let $m \in \frac{1}{T} \mathbb{Z}_{+}$. Recall from Theorem 2.5 (8) and Proposition 4.2 that the linear map $\phi: \quad V \rightarrow V$ defined by $\phi(v)=e^{L(1)}(-1)^{L(0)} v$ for $v \in V$ induces an anti-isomorphism from $A_{g^{-1}, m}(V)$ to $A_{g, m}(V)$ and a linear isomorphism from $A_{g^{-1}, n, m}(V)$ to $A_{g, m, n}(V)$ such that $\phi\left(a *_{g^{-1}, m, p}^{n} b\right)=\phi(b) * m{ }_{g, n, p}^{m} \phi(a)$, for $a \in$ $A_{g^{-1}, n, p}(V), b \in A_{g^{-1}, p, m}(V), p \in \frac{1}{T} \mathbb{Z}_{+}$.

Let $U$ be an $A_{g, m}(V)$-module and $U^{*}$ the dual space of $U$. Then $U^{*}$ is an $A_{g^{-1}, m}(V)$-module with the following action:

$$
(u \cdot f)(x)=f(\phi(u) \cdot x)=(f, \phi(u) \cdot x),
$$

for $u \in A_{g^{-1}, m}(V), f \in U^{*}$ and $x \in U$.

Now let

and

$$
M(U)=\bigoplus_{n \in \frac{1}{T} \mathbb{Z}_{+}} A_{g, n, m}(V) \otimes_{A_{g, m}(V)} U
$$

$$
M\left(U^{*}\right)=\bigoplus_{n \in \frac{1}{T} \mathbb{Z}_{+}} A_{g^{-1}, n, m}(V) \otimes_{A_{g^{-1}, m}(V)} U^{*}
$$

be the Verma type admissible $g$-twisted and $g^{-1}$-twisted $V$-modules generated by $U$ and $U^{*}$ respectively. We define a bilinear pairing $(\cdot, \cdot)$ on $M\left(U^{*}\right) \times M(U)$ as follows:

$$
(x \otimes f, y \otimes u)=\left(f,\left[\left(\phi(x) *_{g, m, n}^{m} y\right)\right] \cdot u\right),
$$

for $x \in A_{g^{-1}, n, m}(V), y \in A_{g, n, m}(V), f \in U^{*}, u \in U, n \in \frac{1}{T} \mathbb{Z}_{+}$; and

$$
\left(A_{g^{-1}, p, m}(V) \otimes_{A_{g^{-1}, m}(V)} U^{*}, A_{g, n, m}(V) \otimes_{A_{g, m}(V)} U\right)=0
$$

for $p \neq n$. That is, $\left(M\left(U^{*}\right)(p), M(U)(n)\right)=0$ if $p \neq n$.

As in DJ3 we have

Proposition 6.6. Let $U$ be an $A_{g, m}(V)$-module for $m \in \frac{1}{T} \mathbb{Z}_{+}$. Then

(1) The bilinear pairing $(\cdot, \cdot)$ on $M\left(U^{*}\right) \times M(U)$ is well defined and is invariant in the sense that

$$
\left(Y_{M\left(U^{*}\right)}(u, z) w^{\prime}, w\right)=\left(w^{\prime}, Y_{M(U)}\left(e^{z L(1)}\left(-z^{-2}\right)^{L(0)} u, z^{-1}\right) w\right)
$$


for $w^{\prime} \in M\left(U^{*}\right), w \in M(U)$ and $u \in V$.

(2) The space

$$
J(U)=\left\{w \in M(U) \mid\left(w^{\prime}, w\right)=0, w^{\prime} \in M\left(U^{*}\right)\right\}
$$

is the maximal proper admissible $g$-twisted $V$-submodule of $M(U)$ such that

$$
J(U) \cap M(U)(m)=0 .
$$

In particular, if $U$ is irreducible, then $J(U)$ is the unique maximal proper admissible $g$-twisted submodule of $M(U)$.

(3) Let $V$ be a simple vertex operator algebra such that $A_{g}(V)$ is semisimple. Let $U$ be an irreducible $A_{g}(V)$-module. Then the bilinear pairing $(\cdot, \cdot)$ on $M\left(U^{*}\right) \times M(U)$ is nondegenerate.

The analogue of Theorem 5.3 of DJ3] is the following - the second main theorem in this paper with the similar proof.

Theorem 6.7. Let $V$ be a simple vertex operator algebra and $g$ an automorphism of $V$ of finite order. Then $V$ is g-rational if and only if $A_{g}(V)$ is semisimple and each irreducible admissible $g$-twisted $V$-module is ordinary.

We remark that the condition that each irreducible admissible $g$-twisted $V$ module is ordinary holds for all known simple vertex operator algebras and finite order automorphisms. Although we firmly believe that this is true in general, we cannot prove this in this paper.

\section{REFERENCES}

[B] R. Borcherds, Vertex algebras, Kac-Moody algebras, and the Monster, Proc. Natl. Acad. Sci. USA 83 (1986), 3068-3071. MR843307 (87m:17033)

[DVVV] R. Dijkgraaf, C. Vafa, E. Verlinde and H. Verlinde, The operator algebra of orbifold models, Comm. Math. Phys. 123 (1989), 485-526. MR.1003430 (91c:81132)

[DHVW1] L.J. Dixon, J.A. Harvey, C. Vafa, and E. Witten, Strings on orbifolds, Nucl. Phys. B 261 (1985) 678-686. MR818423 (87k:81104a)

[DHVW2] L.J. Dixon, J.A. Harvey, C. Vafa, and E. Witten, Strings on orbifolds, II, Nucl. Phys. B 274 (1986) 285-314. MR851703 (87k:81104b)

[DGM] L. Dolan, P. Goddard and P. Montague, Conformal field theories, representations and lattice constructions, Comm. Math. Phys. 179 (1996), 61-120. MR.1395218|(97i:81133)

[D] C. Dong, Vertex algebras associated with even lattices, J. Algebra 165 (1994), 90-112. MR.1272580 (95i:17032)

[DJ1] C. Dong and C. Jiang, Bimodules associated to vertex operator algebras, math.QA/ 0601626 .

[DJ2] C. Dong and C. Jiang, Representation theory of vertex operator algebras, Contemp. Math., to appear, math.QA/0603588.

[DJ3] C. Dong and C. Jiang, Rationality of vertex operator algebras, math. QA/0607679.

[DL1] C. Dong and J. Lepowsky, Generalized Vertex Algebras and Relative Vertex Operators, Progress in Math., Vol. 112, Birkhäuser, Boston, 1993. MR1233387 (95b:17032)

[DL2] C. Dong and J. Lepowsky, The algebraic structure of relative twisted vertex operators, J. Pure Appl. Algebra 110 (1996), no. 3, 259-295. MR.1393116 (98e:17036)

[DLM0] C. Dong, H. Li and G. Mason, Compact automorphism groups of vertex operator algebras, Internat. Math. Res. Notices 18 (1996), 913-921. MR1420556 (98a:17044)

[DLM1] C. Dong, H. Li and G. Mason, Regularity of rational vertex operator algebras, Advances in Math. 132 (1997), 148-166. MR.1488241 (98m:17037)

[DLM2] C. Dong, H. Li and G. Mason, Twisted representations of vertex operator algebras, Math. Ann. 310 (1998), 571-600. MR1615132 (99d:17030)

[DLM3] C. Dong, H. Li and G. Mason, Vertex operator algebras and associative algebras, J. Algebra 206 (1998), 67-96. MR1637252 (99i:17029) 
[DLM4] C. Dong, H. Li and G. Mason, Twisted representations of vertex operator algebras and associative algebras, International Math. Research Notices, 8 (1998), 389-397. MR1628239 (99f:17032)

[DLM5] C. Dong, H. Li and G. Mason, Modular invariance of trace functions in orbifold theory and generalized moonshine, Comm. Math. Phys. 214 (2000), 1-56. MR1794264 (2001k:17043)

[DM] C. Dong and G. Mason, On quantum Galois theory, Duke Math. J. 86 (1997), 305-321. MR1430435 (97k:17042)

[DY] C. Dong and G. Yamskulna, Vertex operator algebras, Generalized double and dual pairs, Math. Z. 241 (2002), 397-423. MR1935493(2003j:17038)

[FFR] A. J. Feingold, I. B. Frenkel and J. F. X. Ries, Spinor construction of vertex operator algebras, triality and $E_{8}^{(1)}$, Contemporary Math. 121, 1991. MR.1123265 (92k:17041)

[FHL] I. B. Frenkel, Y. Huang and J. Lepowsky, On axiomatic approaches to vertex operator algebras and modules, Memoirs Amer. Math. Soc. 104, 1993. MR.1142494(94a:17007)

[FLM1] I. B. Frenkel, J. Lepowsky and A. Meurman, Vertex operator calculus, in: Mathematical Aspects of String Theory, Proc. 1986 Conference, San Diego. ed. by S.-T. Yau, World Scientific, Singapore, 1987, 150-188. MR915822

[FLM2] I. B. Frenkel, J. Lepowsky and A. Meurman, Vertex Operator Algebras and the Monster, Pure and Applied Math., Vol. 134, Academic Press, 1988. MR.996026 (90h:17026)

[HMT] A. Hanaki, M. Miyamoto and D. Tambara, Quantum Galois theory for finite groups, Duke Math. J. 97 (1999), 541-544. MR1682988(2000g:17043a)

[L1] J. Lepowsky, Calculus of twisted vertex operators, Proc. Natl. Acad Sci. USA 82 (1985), 8295-8299. MR820716 (88f:17030)

[L2] J. Lepowsky, Perspectives on vertex operators and the Monster, in: Proc. 1987 Symposium on the Mathematical Heritage of Hermann Weyl, Duke Univ., Proc. Symp. Pure. Math., American Math. Soc. 48 (1988), 181-197. MR974335 (90f:17031)

[LL] J. Lepowsky and H. Li, Introduction to Vertex Operator Algebras and Their Representations, Progress in Mathematics, Vol. 227, Birkhäuser Boston, Inc., Boston, MA, 2004. MR2023933 (2004k:17050)

[MT] M. Miyamoto and K. Tanabe, Uniform product of $A_{g, n}(V)$ for an orbifold model $V$ and $G$-twisted Zhu algebra, J. Algebra 274 (2004), 80-96. MR2040864(2005d:17037)

[Z] Y. Zhu, Modular invariance of characters of vertex operator algebras, J. Amer. Math. Soc. 9 (1996), 237-302. MR1317233 (96c:17042)

Department of Mathematics, University of California, Santa Cruz, California 95064

Department of Mathematics, Shanghai Jiaotong University, Shanghai 200030, PeoPLE'S Republic of ChinA 